\title{
Uniform-type structures on lattice-valued spaces and frames
}

\author{
Javier Gutiérrez García ${ }^{\mathrm{a}}$, Iraide Mardones-Pérez ${ }^{\mathrm{a}}$, Jorge Picado ${ }^{\mathrm{b}, *}$, \\ María Angeles de Prada Vicente ${ }^{a}$ \\ ${ }^{a}$ Departamento de Matemáticas, Universidad del País Vasco-Euskal Herriko Unibertsitatea, Apartado 644, 48080, Bilbao, Spain \\ ${ }^{\mathrm{b}}$ CMUC, Department of Mathematics, University of Coimbra, Largo D. Dinis, P-3001 454 Coimbra, Portugal
}

Received 28 June 2007; received in revised form 6 March 2008; accepted 6 March 2008

\begin{abstract}
By introducing lattice-valued covers of a set, we present a general framework for uniform structures on very general $L$-valued spaces (for $L$ an integral commutative quantale). By showing, via an intermediate $L$-valued structure of uniformity, how filters of covers may describe the uniform operators of Hutton, we prove that, when restricted to Girard quantales, this general framework captures a significant class of Hutton's uniform spaces. The categories of $L$-valued uniform spaces and $L$-valued uniform frames here introduced provide (in the case $L$ is a complete chain) the missing vertices in the commutative cube formed by the classical categories of topological and uniform spaces and their corresponding pointfree counterparts (forming the base of the cube) and the corresponding $L$-valued categories (forming the top of the cube).
\end{abstract}

(C) 2008 Elsevier B.V. All rights reserved.

Keywords: Frame; Locale; Quantale; Girard quantale; Integral commutative quantale; $L$-valued space; $L$-valued frame; Uniformity; Cover; Entourage; Uniform operator; Galois connection; Axiality; Polarity

\section{Introduction}

In classical topology, uniform structures are usually approached in terms of covers [33], via double powersets of the form $2^{2^{X}}$, or, equivalently, in terms of entourages [34], via powersets of the form $2^{X \times X}$. Entourages, being binary relations, may be also easily described by polarities (that is, Galois connections between power sets) and axialities (that is, residuated pairs between power sets). This gives two more equivalent descriptions of uniform spaces.

One of the questions of interest in lattice-valued topology concerns well-founded definitions of uniform-type structures (see the introduction to [26]). The uniform structures of Hutton [15] have been regarded as the lattice-valued counterpart to the classical covering uniformities of Tukey (see [29]), in opposition to the fuzzy uniform spaces of Lowen [20], which are an extension of the entourage approach of Weil. It is well-known that, opposite to the classical case, these two notions are not equivalent. In this paper we show that residuations rather than covers are the root of Hutton's approach, and how lattice-valued covers of a set may describe lattice-valued uniformities. This approach leads to a new category of $L$-valued uniform spaces in which a significant class of Hutton uniform spaces fits nicely. It will be apparent that this category is the natural generalization of the classical Tukey's definition to the $L$-valued context.

\footnotetext{
* Corresponding author. Tel.: +351 239791155.

E-mail addresses: javier.gutierrezgarcia@ehu.es (J. Gutiérrez García), iraide.mardones@ehu.es (I. Mardones-Pérez), picado@mat.uc.pt (J. Picado), mariangeles.deprada@ehu.es (M.A. de Prada Vicente).
} 
Some interesting observations about Hutton's definition will appear along the way. Namely, it will be clear why and when Hutton's axiomatization, based on residuated maps (axialities [5]), can be equivalently seen as an $L$-residuated uniformity. Also, an open question appears: what happens if Hutton's axiomatization is formulated in terms of Galois maps (polarities [5]), generalizing the meaning of being a reflexive relation (an entourage) in this contravariant case?

The motivation for this paper arose from an observation of Pultr and Rodabaugh in [26] that lattice-valued frames may be useful in the establishment of well-founded definitions of uniform-type structures. To put this in perspective, let us recall that a frame is a complete lattice $M$ satisfying the infinite distributivity law

$$
m \wedge \bigvee s=\bigvee\{m \wedge s \mid s \in S\}
$$

for all $m \in M$ and $S \subseteq M$, and a frame homomorphism $h: M \rightarrow N$ is a map preserving finite meets (including the top 1) and arbitrary joins (including the bottom 0 ). The resulting category will be denoted by Frm. The two-element frame $\{0<1\}$ will be denoted by 2 .

If $X$ is a topological space, the lattice $\mathcal{O}(X)$ of its open sets is a frame, and if $f: X \rightarrow Y$ is a continuous map then $\mathcal{O}(f): \mathcal{O}(Y) \rightarrow \mathcal{O}(X)$ defined by $\mathcal{O}(f)(U)=f^{-1}(U)$ is a frame homomorphism. Thus, we have a contravariant functor $\mathcal{O}:$ Top $\rightarrow$ Frm. Finally, recall the standard spectrum construction

$$
\Sigma(M)=\left(\{p: M \rightarrow 2 \mid p \in \operatorname{Frm}\},\left\{\Sigma_{m} \mid m \in M\right\}\right)
$$

for a frame $M$ (where $\Sigma_{m}=\{p \mid p(m)=1\}$ ). Defining, for each frame homomorphism $h: M \rightarrow N, \Sigma(h): \Sigma(N) \rightarrow$ $\Sigma(M)$ by $\Sigma(h)(p)=p \cdot h$, this constitutes a contravariant functor $\Sigma:$ Frm $\rightarrow$ Top. The following are well-known facts: $\Sigma$ is a right adjoint for $\mathcal{O}$, each $\Sigma(M)$ is a sober space (i.e. a $T_{0}$ space $X$ whose only meet irreducible elements are $X \backslash \overline{\{x\}}$ ), the unit map $X \rightarrow \Sigma \mathcal{O}(X)$ is a homeomorphism if and only if $X$ is sober, and the unit homomorphism $\mathcal{O} \Sigma(M) \rightarrow M$ is an isomorphism if and only if $M$ is spatial i.e. isomorphic to the frame of open sets of some topological space $X$. For more details about frames see, e.g. [17] or [23].

The above dual adjunction $\mathcal{O} \dashv \Sigma$ between the category of frames and the category of topological spaces can be easily adapted to the uniform setting, yielding a dual adjunction between the category UFrm of uniform frames (introduced by Isbell [16], and studied in detail by Pultr [25] in terms of covers; for information about other different ways of describing them see [6,22]) and the category Unif of uniform spaces of Weil [34] and Tukey [33]. Then, denoting by $F_{1}$ and $F_{2}$, respectively, the forgetful functors Unif $\rightarrow$ Top and UFrm $\rightarrow$ Frm forgetting the uniform structure, the diagram

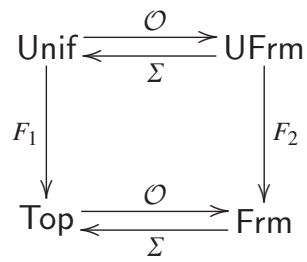

commutes.

In [26], the authors introduced $L$-valued frames, for a linearly ordered $L$, which relate to frames in a way parallel to that in which the $l_{L}$ functor (see $[19,18]$ ) relates $L$-valued topological spaces to topological spaces. There is a dual adjunction between $L$-Top and $L$-Frm that shows that $L$-valued frames generalize $L$-valued topological spaces in a way parallel to frames generalizing topological spaces. Specifically, denoting by $\chi_{L}^{T}$ and $\chi_{L}^{F}$ the (characteristic) functors embedding the categories of 2 -valued objects in question in the corresponding categories of $L$-valued objects (see Section 6 for a description of the embedding functor $\chi_{L}^{F}$ ), the diagram

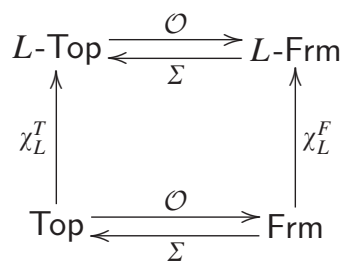


commutes. Putting it together with (1.1) we get an incomplete diagram:

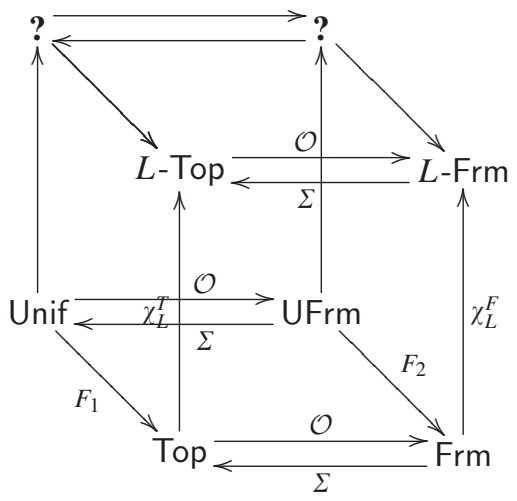

A natural question arises:

Does there exist two types of structure (i.e. appropriate notions of L-valued uniform spaces and frames) that would allow us to complete the cube (by filling in the two question marks) in such a way that the two new vertical arrows also represent embedding functors, that the two new diagonal arrows also represent forgetful functors, that the new horizontal arrows also establish an adjunction, and that the whole diagram commutes?

The answer is not immediately obvious: as the authors of [26] point out, the direct approach through uniformizing the $L$-topology $\tau$ as a frame is not satisfactory. Indeed, a uniformity on $\tau$ induces a uniformity on the lattice $L$ of values (as observed by Banaschewski-see [26]); so, when $L$ is linear, we would stay within the crisp case, since the only linearly ordered frame admitting a uniformity is the two-element frame $2=\{0<1\}$.

It is our purpose in this paper to show that all the points raised above can be addressed in a satisfactory way. We introduce categories $L$-Unif and $L$-UFrm, for $L$ a general strictly two-sided commutative quantale and a complete chain, respectively, that fill in the two question marks: they are related, respectively, to the categories $L$-Top and $L$-Frm in a parallel way and again, when $L$ is a linearly ordered complete lattice, $L$-UFrm generalizes $L$-Unif in a way parallel to uniform frames generalizing uniform spaces. Then we present an equivalent presentation for $L$-Unif in terms of residuated mappings that will encompass Hutton's original definition whenever $L$ is a Girard quantale. It will be apparent that a slight change in one of the axioms of Hutton makes a big difference and allows the extension of the definition to more general contexts for a certain class of Hutton uniform spaces.

The paper is organized as follows. We begin, in Section 2, by establishing some notations and by recalling some background on $L$-topological spaces and uniform frames. Then, in Section 3, we introduce $L$-valued covers and use them to axiomatize $L$-valued uniform structures for very general lattices $L$ (namely, strictly two-sided commutative quantales). In Section 4 we give an alternative, equivalent, formulation in terms of residuated mappings. In Section 5, we show that the latter approach, when restricted to Girard quantales, implies Hutton's axiomatization. Several arguments are presented in favor of our approach. After recalling, in Section 6, some basic facts about $L$-valued frames we relate, in Section 7, $L$-valued uniform spaces with uniform spaces and provide in Section 8 the missing vertex for the above cube: the notion of an $L$-valued uniform frame.

\section{Preliminaries and notation}

\subsection{L-valued spaces}

We recall that $(L, \leqslant, *)$ is a quantale if

(1) $(L, \leqslant)$ is a complete lattice (with top element $1=\bigwedge \emptyset$ and bottom $0=\bigvee \emptyset$ ).

(2) $*$ is an associative binary operation distributive over arbitrary joins:

$$
\alpha *\left(\bigvee_{i \in I} \beta_{i}\right)=\bigvee_{i \in I}\left(\alpha * \beta_{i}\right)
$$


Since the operators $\alpha *(-)$ preserve arbitrary joins, every quantale is residuated, i.e. there exist the corresponding right adjoints $\alpha \rightarrow(-)$ defined by the relation

$$
\alpha * \beta \leqslant \gamma \Longleftrightarrow \beta \leqslant \alpha \rightarrow \gamma
$$

In particular, $\rightarrow$ is given by $\alpha \rightarrow \gamma=\bigvee\{\beta \in L \mid \alpha * \beta \leqslant \gamma\}$. A quantale $(L, \leqslant, *$ ) is strictly two-sided (or simply integral) if

(3) $(L, *)$ is a monoid whose unit is the top element 1 .

(Notice that an integral quantale is an integral $c l$-monoid in the sense of [12].)

We list here some of the basic properties of integral quantales needed in the sequel:

(Q1) $\beta \leqslant \gamma \Rightarrow \alpha * \beta \leqslant \alpha * \gamma$.

(Q2) $\alpha * 0=0 * \alpha=0$ for all $\alpha \in L$.

(Q3) $\alpha \leqslant(\alpha \rightarrow 0) \rightarrow 0$ for all $\alpha \in L$.

Sometimes integral commutative quantales are referred to as complete residuated lattices. Any frame is automatically a commutative integral quantale (where the binary operation $*$ is given by the meet $\wedge$ ).

A commutative unital quantale (i.e. a commutative quantale with a unit $\varepsilon$ satisfying $\alpha * \varepsilon=\varepsilon * \alpha=\alpha$ for all $\alpha$ ) is called a Girard quantale if it satisfies the law of double negation

(Q4) $\alpha=(\alpha \rightarrow 0) \rightarrow 0$ for all $\alpha \in L$.

Note that a Girard quantale is necessarily integral since

$$
\varepsilon \rightarrow 0=\bigvee\{\alpha \in L \mid \varepsilon * \alpha \leqslant 0\}=0
$$

and, consequently, $\varepsilon=(\varepsilon \rightarrow 0) \rightarrow 0=0 \rightarrow 0=1$.

From now on, except when otherwise stated, we will assume that $L$ is an integral commutative quantale. For any set $X$, the set $L^{X}$ of mappings $X \rightarrow L$, with the partial order

$$
a \leqslant b \equiv a(x) \leqslant b(x) \text { for each } x \in X
$$

is also an integral quantale: joins, meets and the binary operation $*$ are just defined pointwisely. If $A \subset X$, then $1_{A} \in L^{X}$ denotes the characteristic function of $A$, hence we denote the top element of $L^{X}$ by $1_{X}$ and the bottom by $1_{\emptyset}$. The constant member of $L^{X}$ with value $\alpha$ is denoted $\alpha$ too. Given a map $f: X \rightarrow Y, a \in L^{X}$ and $b \in L^{Y}$, the usual (Zadeh) image and preimage operators are defined as follows:

$$
f^{\rightarrow}(a)=\bigvee_{x \in X} a(x) \wedge 1_{\{f(x)\}},
$$

and

$$
f^{\leftarrow}(b)=b \cdot f
$$

the composition of $f$ and $b$. We shall need a number of properties of the operators just defined viz.:

Properties 2.1. Let $f: X \rightarrow Y, g: Y \rightarrow Z, a \in L^{X}, b, b_{1}, b_{2} \in L^{Y}, c \in L^{Z}$. Then:

(1) $f^{\leftarrow}$ preserves sups and infs; $f^{\rightarrow}$ preserves sups (in particular, they are both order-preserving),

(2) $a \leqslant f \leftarrow(f \rightarrow(a))$,

(3) $f^{\rightarrow}\left(f^{\leftarrow}(b)\right) \leqslant b$, and $f^{\rightarrow}\left(f^{\leftarrow}(b)\right)=b$ if and only if $f$ is surjective,

(4) $(g \cdot f) \rightarrow(a)=g \rightarrow(f \rightarrow(a))$,

(5) $(g \cdot f) \leftarrow(c)=g^{\leftarrow}\left(f^{\leftarrow}(c)\right)$

(6) $f^{\leftarrow}\left(b_{1} * b_{2}\right)=f^{\leftarrow}\left(b_{1}\right) * f \leftarrow\left(b_{2}\right)$, and

(7) $f^{\rightarrow}(a) * b \neq 1_{\emptyset}$ if and only if $a * f^{\leftarrow}(b) \neq 1_{\emptyset}$. 
An $L$-valued topological space $[4,14]$ (shortly, an $L$-topological space) is a pair $(X, \tau)$ consisting of a set $X$ and a subset $\tau$ of $L^{X}$ (the $L$-valued topology or $L$-topology on the set $X$ ), containing $1_{\varnothing}$ and $1_{X}$ and closed under finite meets and arbitrary joins.

Given two L-valued topological spaces $\left(X, \tau_{1}\right),\left(Y, \tau_{2}\right)$ a map $f: X \rightarrow Y$ is an L-continuous map if the correspondence $b \mapsto f^{\leftarrow}(b)$ maps $\tau_{2}$ into $\tau_{1}$. The resulting category will be denoted by $L$-Top.

Of course, when $L=2$, an $L$-topological space is precisely a topological space and there is an isomorphism between Top and $L$-Top, via the characteristic functor (the one associating to each subset its characteristic function and leaving morphisms unchanged). If $L$ is a frame then the $L$-topologies, being subframes of the frame $L^{X}$, are frames as well.

\subsection{Uniform spaces}

There are several equivalent axiomatizations of the notion of uniformity on a set $X$. We will refer to the one introduced by Tukey [33] in which the basic term is the one of uniform cover of $X$. A cover $\mathcal{U}$ refines a cover $\mathcal{V}$, and in this case one writes $\mathcal{U} \preccurlyeq \mathcal{V}$, if for each $U \in \mathcal{U}$ there exists $V \in \mathcal{V}$ such that $U \subseteq V$. For each cover $\mathcal{U}$ of $X$ and $A \subseteq X$, let $\operatorname{st}(A, \mathcal{U}):=\bigcup\{U \in \mathcal{U} \mid U \cap A \neq \emptyset\}$ be the $\operatorname{star}$ of $A$ in $\mathcal{U}$ and $\operatorname{st}(\mathcal{U}):=\{\operatorname{st}(V, \mathcal{U}) \mid V \in \mathcal{U}\}$, which is a cover too. A uniformity on $X$ is a set $\mu$ of covers of $X$ such that:

(U1) $\mu$ is a filter in the preordered set $(\operatorname{Cov}(X), \preccurlyeq)$ of all covers of $X$.

(U2) for each $\mathcal{U} \in \mu$ there is some $\mathcal{V} \in \mu$ such that the $\operatorname{cover} \operatorname{st}(\mathcal{V})$ refines $\mathcal{U}$.

A map $f:(X, \mu) \longrightarrow(Y, v)$ between uniform spaces is uniformly continuous if for every $\mathcal{V} \in v, f^{-1}[\mathcal{V}]=\left\{f^{-1}(V) \mid\right.$ $V \in \mathcal{V}\}$ belongs to $\mu$. We denote the resulting category of uniform spaces and uniformly continuous maps by Unif.

The uniform topology $\mathcal{T}_{\mu}$ induced by $(X, \mu)$ is the one generated by the neighborhood basis $\{\operatorname{st}(\{x\}, \mathcal{U}) \mid \mathcal{U} \in \mu\}$ for each $x \in X$. The correspondence $(X, \mu) \rightarrow\left(X, \mathcal{T}_{\mu}\right)$ defines the forgetful functor $F_{1}:$ Unif $\rightarrow$ Top.

\subsection{Uniform frames}

Tukey's approach to uniform spaces via covers was the first to be studied in the pointfree context of frames. In [16] Isbell introduced uniformities on frames, as the precise translation into frame terms of Tukey's notion, later developed in detail by Pultr [25]. We note that, as in the case of spaces, there are several different ways of describing uniformities on frames, such as the functional uniformities of Fletcher-Hunsaker ([7], [8]) and the entourage unifromities of Picado [21].

Let $M$ be a frame. A set $C \subseteq M$ is a cover of $M$ if $\bigvee C=1$. The set of all covers of $M$, denoted as $\operatorname{Cov}(M)$, can be preordered as follows: a cover $C$ refines a cover $D$, written $C \preccurlyeq D$, if for each $c \in C$ there is some $d \in D$ with $c \leqslant d$. Thus, $(\operatorname{Cov}(M), \preccurlyeq)$ is a preordered set with meets and joins: take for $C \wedge D$ the cover $\{c \wedge d \mid c \in C, d \in D\}$ and for $C \vee D$ just the union $C \cup D$.

For each $m \in M$, the $\operatorname{star}$ of $m$ in $C$ is the element st $(m, C):=\bigvee\{c \in C \mid c \wedge m \neq 0\}$ and $\operatorname{st}(C):=\{\operatorname{st}(c, C) \mid c \in C\}$ which is also a cover of $M$. Further, for each family $\mathcal{C}$ of covers of $M$, let

$n \stackrel{\mathcal{C}}{\triangleleft} m$ if there is $C \in \mathcal{C}$ such that $\operatorname{st}(n, C) \leqslant m$, for any $n, m \in M$.

A family $\mathcal{C}$ of covers of a frame $M$ is a uniformity (see, for example, [2]; the notion of a uniform frame goes back to Pultr [25]), provided that:

(U1) $\mathcal{C}$ is a filter in the preordered set $(\operatorname{Cov}(M), \preccurlyeq)$.

(U2) For each $C \in \mathcal{C}$ there is a $D \in \mathcal{C}$ such that the cover st $(D)$ refines $C$.

(U3) For every $m \in M, m=\bigvee\{n \in M \mid n \stackrel{\mathcal{C}}{\triangleleft} m\}$.

Note that property (U3) is equivalent to the regularity of the frame $M$, see [25, Theorem 2.8].

The pair $(M, \mathcal{C})$ is then called a uniform frame. Let $(M, \mathcal{C})$ and $(N, \mathcal{D})$ be uniform frames. A frame homomorphism $h: M \rightarrow N$ is a uniform homomorphism if, for every $C \in \mathcal{C}, h[C]=\{h(c) \mid c \in C\} \in \mathcal{D}$. We denote by UFrm the category of uniform frames and uniform homomorphisms. This category is related to the category Unif of uniform spaces and uniformly continuous maps by a dual adjunction via the open and spectrum (contravariant) functors: 
The open functor $\mathcal{O}:$ Unif $\rightarrow$ UFrm assigns to each uniform space $(X, \mu)$ the uniform frame $\left(\mathcal{T}_{\mu}, \mathcal{C}_{\mathcal{T}_{\mu}}\right)$, where $\mathcal{T}_{\mu}$ is the topology induced by $\mu$ and $\mathcal{C}_{\mathcal{T}_{\mu}}$ is the collection of all $\mathcal{T}_{\mu}$-open covers of $\mathcal{T}_{\mu}$. If $f:(X, \mu) \rightarrow(Y, v)$ is uniformly continuous, then $\mathcal{O}(f): \mathcal{O}(Y, v) \rightarrow \mathcal{O}(X, \mu)$ defined, for each $V \in \mathcal{T}_{v}$, by $\mathcal{O}(f)(V)=f^{-1}(V)$, is a uniform homomorphism.

On the other hand, the spectrum functor $\Sigma:$ UFrm $\rightarrow$ Unif assigns to each uniform frame $(M, \mathcal{C})$ the uniform space ( $\Sigma M, \mu_{\Sigma M}$ ), being $\Sigma M=\{p: M \rightarrow 2 \mid p \in \mathrm{Frm}\}$ the set of points of $M$ and $\mu_{\Sigma M}$ the filter of covers of $\Sigma M$ generated by $\left(\left\{\Sigma_{m} \mid m \in C\right\}\right)_{C \in \mathcal{C}}$, where $\Sigma_{m}=\{p \in \Sigma M \mid p(m)=1\}$. If $h:(M, \mathcal{C}) \rightarrow(N, \mathcal{D})$ is a uniform homomorphism then $\Sigma(h): \Sigma(N, \mathcal{D}) \rightarrow \Sigma(M, \mathcal{C})$, given by $\Sigma(h)(q)=q \cdot h$, is uniformly continuous.

This adjunction makes the diagram in (1.1) commutative.

\section{Covering $L$-valued uniform spaces}

We say that $\mathcal{A} \subseteq L^{X}$ is an $L$-cover of $X$ if $\bigvee \mathcal{A}=1_{X}$. For any $\mathcal{A}, \mathcal{B} \subseteq L^{X}$ we write $\mathcal{A} \preccurlyeq \mathcal{B}$ if for each $a \in \mathcal{A}$ there exists $b \in \mathcal{B}$ such that $a \leqslant b$.

The relation just defined makes the set of all $L$-covers of $X$, noted as $L-\operatorname{Cov}(X)$, a preordered set. Let $\mathcal{A} * \mathcal{B}=\{a * b \mid$ $a \in \mathcal{A}, b \in \mathcal{B}\}$, where $a * b: X \rightarrow L$ is defined pointwisely and $\mathcal{A} \wedge \mathcal{B}=\{a \wedge b \mid a \in \mathcal{A}, b \in \mathcal{B}\}$. Clearly, $\mathcal{A} * \mathcal{B} \preccurlyeq \mathcal{A} \wedge \mathcal{B}$, since $a * b \leqslant a * 1_{X}=a$ and $a * b \leqslant 1_{X} * b=b$.

Proposition 3.1. For every $L$-covers $\mathcal{A}$ and $\mathcal{B}, \mathcal{A} * \mathcal{B}$ and $\mathcal{A} \wedge \mathcal{B}$ are $L$-covers of $X$.

Proof. Since $*$ distributes over arbitrary joins,

$$
\bigvee(\mathcal{A} * \mathcal{B})=\bigvee \mathcal{A} * \bigvee \mathcal{B}=1_{X} * 1_{X}=1_{X},
$$

and it follows that $\mathcal{A} * \mathcal{B}$ is an $L$-cover. Then $\mathcal{A} \wedge \mathcal{B}$ is also an $L$-cover because $\mathcal{A} * \mathcal{B} \preccurlyeq \mathcal{A} \wedge \mathcal{B}$.

Notice that, since $\mathcal{A} \wedge \mathcal{B}$ is an $L$-cover, then $\mathcal{A} \wedge \mathcal{B}$ is the infimum of $\mathcal{A}$ and $\mathcal{B}$ in the preordered set $(L-\operatorname{Cov}(X), \preccurlyeq)$ of all $L$-covers of $X$.

For each $a \in L^{X}$ and $\mathcal{A} \subseteq L^{X}$, let

$$
\operatorname{st}(a, \mathcal{A}):=\bigvee\left\{b \in \mathcal{A} \mid b * a \neq 1_{\emptyset}\right\}
$$

and

$$
\operatorname{st}(\mathcal{A}):=\{\operatorname{st}(a, \mathcal{A}) \mid a \in \mathcal{A}\},
$$

which is an $L$-cover (whenever $\mathcal{A}$ is a cover).

Proposition 3.2. Let $\mathcal{A}, \mathcal{B} \subseteq L^{X}$ and $a, b \in L^{X}$. Then:

(1) If $\mathcal{A}$ is an L-cover then $a \leqslant \operatorname{st}(a, \mathcal{A})$ and, consequently, $\mathcal{A} \preccurlyeq \operatorname{st}(\mathcal{A})$.

(2) If $a \leqslant b$ then $\operatorname{st}(a, \mathcal{A}) \leqslant \operatorname{st}(b, \mathcal{A})$.

(3) If $\mathcal{A} \preccurlyeq \mathcal{B}$ then $\operatorname{st}(a, \mathcal{A}) \leqslant \operatorname{st}(a, \mathcal{B})$.

(4) $\operatorname{st}(\bigvee \mathcal{B}, \mathcal{A})=\bigvee_{b \in \mathcal{B}} \operatorname{st}(b, \mathcal{A})$.

(5) If $\mathcal{A}$ is an $L$-cover then $\operatorname{st}(\operatorname{st}(a, \mathcal{A}), \mathcal{A}) \leqslant \operatorname{st}(a, \operatorname{st}(\mathcal{A}))$.

(6) Let $f: X \rightarrow Y, \mathcal{B} \subseteq L^{Y}, f^{-1}[\mathcal{B}]:=\left\{f^{\leftarrow}(b) \mid b \in \mathcal{B}\right\}$ and $c \in L^{Y}$. Then, $\operatorname{st}\left(f^{\leftarrow}(c), f^{-1}[\mathcal{B}]\right) \leqslant f^{\leftarrow}(\operatorname{st}(c, \mathcal{B}))$.

Proof. (1) $a=1_{X} * a=\left(\bigvee_{b \in \mathcal{A}} b\right) * a=\bigvee\left\{b * a \mid b \in \mathcal{A}, b * a \neq 1_{\varnothing}\right\} \leqslant \operatorname{st}(a, \mathcal{A})$. (2,3) These are obvious.

$$
\begin{aligned}
\operatorname{st}(\bigvee \mathcal{B}, \mathcal{A}) & =\bigvee\left\{a \in \mathcal{A} \mid a * \bigvee \mathcal{B} \neq 1_{\varnothing}\right\}=\bigvee\left\{a \in \mathcal{A} \mid \bigvee_{b \in \mathcal{B}}(a * b) \neq 1_{\emptyset}\right\} \\
& =\bigvee\left\{a \in \mathcal{A} \mid a * b \neq 1_{\emptyset} \text { for some } b \in \mathcal{B}\right\}=\bigvee_{b \in \mathcal{B}} \operatorname{st}(b, \mathcal{A}) .
\end{aligned}
$$


(5) For each $a \in L^{X}$ we have, using the previous property,

$$
\operatorname{st}(\operatorname{st}(a, \mathcal{A}), \mathcal{A})=\bigvee\left\{\operatorname{st}(b, \mathcal{A}) \mid b \in \mathcal{A}, b * a \neq 1_{\emptyset}\right\} .
$$

Since, for each such $b, \operatorname{st}(b, \mathcal{A}) \in \operatorname{st}(\mathcal{A})$ and $\operatorname{st}(b, \mathcal{A}) * a \geqslant b * a \neq 1 \emptyset$, we have immediately $\operatorname{st}(\operatorname{st}(a, \mathcal{A}), \mathcal{A}) \leqslant \operatorname{st}(a, \operatorname{st}(\mathcal{A}))$.

(6) Since $f^{\leftarrow}$ preserves arbitrary sups and the binary operation $*$ (see Properties 2.1), we have:

$$
\begin{aligned}
\operatorname{st}\left(f^{\leftarrow}(c), f^{-1}[\mathcal{B}]\right) & =\bigvee\left\{f^{\leftarrow}(b) \mid b \in \mathcal{B}, f^{\leftarrow}(b) * f^{\leftarrow}(c) \neq 1_{\varnothing}\right\}=f^{\leftarrow}\left(\bigvee\left\{b \in \mathcal{B} \mid f^{\leftarrow}(b * c) \neq 1_{\varnothing}\right\}\right) \\
& \leqslant f^{\leftarrow}\left(\bigvee\left\{b \in \mathcal{B} \mid b * c \neq 1_{\varnothing}\right\}\right)=f^{\leftarrow}(\operatorname{st}(c, \mathcal{B})) .
\end{aligned}
$$

Definition 3.3. We say that a pair $(X, \mathfrak{U})$ consisting of a set $X$ and a nonempty family $\mathfrak{U}$ of $L$-covers of $X$ is a covering $L$-uniform space whenever the following conditions are satisfied:

(C1) $\mathcal{A} \preccurlyeq \mathcal{B}, \mathcal{A} \in \mathfrak{U} \Rightarrow \mathcal{B} \in \mathfrak{U}$.

(C2) For every $\mathcal{A}, \mathcal{B} \in \mathfrak{U}, \mathcal{A} \wedge \mathcal{B} \in \mathfrak{U}$.

(C3) For each $\mathcal{A} \in \mathfrak{U}$ there exists $\mathcal{B} \in \mathfrak{U}$ such that $\operatorname{st}(\mathcal{B}) \preccurlyeq \mathcal{A}$.

A base for the covering $L$-uniformity $\mathfrak{U}$ is any subcollection of $\mathfrak{U}$ from which $\mathfrak{U}$ can be recovered by applying condition $(\mathrm{C} 1)$.

A map $f:(X, \mathfrak{U}) \rightarrow(Y, \mathfrak{B})$ is a uniform homomorphism if, for every $\mathcal{B} \in \mathfrak{B}, f^{-1}[\mathcal{B}] \in \mathfrak{U}$. The resulting category will be denoted by $L$-Unif. Of course, for $L=2$, this is precisely the category of (covering) uniform spaces of Tukey [33].

For each $(X, \mathfrak{U}) \in L$-Unif define

$$
\tau_{\mathfrak{U}}:=\left\{a \in L^{X} \mid a=\bigvee\left\{b \in L^{X} \mid \operatorname{st}(b, \mathcal{A}) \leqslant a \text { for some } \mathcal{A} \in \mathfrak{U}\right\}\right\} .
$$

Note that, when $L=2, \tau_{\mathfrak{U}}$ is just the crisp topology induced by the (classical) uniformity $\mathfrak{U}$ on $X$.

Proposition 3.4. $\left(X, \tau_{\mathfrak{U}}\right)$ is an L-topological space whenever $L$ is a frame.

Proof. It suffices to check that int : $L^{X} \rightarrow L^{X}$ defined by

$$
\operatorname{int}(a)=\bigvee\left\{b \in L^{X} \mid \operatorname{st}(b, \mathcal{A}) \leqslant a \text { for some } \mathcal{A} \in \mathfrak{U}\right\}
$$

is an $L$-interior operator [14], that is:

(I1) $\operatorname{int}\left(1_{X}\right)=1_{X}$.

(I2) $\operatorname{int}(a) \leqslant a$ for every $a \in L^{X}$.

(I3) $\operatorname{int}(\operatorname{int}(a))=\operatorname{int}(a)$ for every $a \in L^{X}$.

(I4) $\operatorname{int}(a \wedge b)=\operatorname{int}(a) \wedge \operatorname{int}(b)$ for every $a, b \in L^{X}$.

(I1) and (I2) are trivially satisfied.

(I3) Let $b \in L^{X}$ satisfying $\operatorname{st}(b, \mathcal{A}) \leqslant a$ for some $\mathcal{A} \in \mathfrak{U}$ and take $\mathcal{B} \in \mathfrak{U}$ such that $\operatorname{st}(\mathcal{B}) \preccurlyeq \mathcal{A}$. Then $\operatorname{st}(b, \mathcal{B}) \leqslant \operatorname{int}(a)$ since $\operatorname{st}(\operatorname{st}(b, \mathcal{B}), \mathcal{B}) \leqslant \operatorname{st}(b, \operatorname{st}(\mathcal{B})) \leqslant \operatorname{st}(b, \mathcal{A}) \leqslant a$ 4position 3.2. This shows that $\operatorname{int}(a) \leqslant \operatorname{int}(\operatorname{int}(a))$ and the equality follows from (I2).

(I4) Since $L$ is a frame, meets distribute over arbitrary joins and then (I4) follows immediately from (C2).

$\tau_{\mathfrak{U}}$-open $L$-covers of $\mathfrak{U}$ form a base for the covering $L$-uniformity $\mathfrak{U}$ :

Proposition 3.5. If $\mathcal{A} \in \mathfrak{U}$ then $\operatorname{int}(\mathcal{A}):=\{\operatorname{int}(a) \mid a \in \mathcal{A}\} \in \mathfrak{U}$.

Proof. Let $\mathcal{B} \in \mathfrak{U} \operatorname{such}$ that $\operatorname{st}(\mathcal{B}) \preccurlyeq \mathcal{A}$. Then $\mathcal{B} \preccurlyeq \operatorname{int}(\mathcal{A})$. Indeed, for each $b \in \mathcal{B}, b \leqslant \operatorname{st}(b, \mathcal{B}) \leqslant a$ for some $a \in \mathcal{A}$, which shows that $b \leqslant \operatorname{int}(a)$. 
For any $a, b \in L^{X}$ we write $b \stackrel{\mathfrak{U}}{\triangleleft} a$ whenever $\operatorname{st}(b, \mathcal{A}) \leqslant a$ for some $\mathcal{A} \in \mathfrak{U}$. The following property of the $L$-topology $\tau_{\mathfrak{U}}$ follows immediately from the previous proposition.

Corollary 3.6. For every $a \in \tau_{\mathfrak{U}}, a=\bigvee\left\{b \in \tau_{\mathfrak{U}} \mid b \stackrel{\mathfrak{U}}{\triangleleft} a\right\}$.

Proposition 3.7. For any $f:(X, \mathfrak{U}) \rightarrow(Y, \mathfrak{B})$ in $L$-Unif, $f:\left(X, \tau_{\mathfrak{U}}\right) \rightarrow\left(Y, \tau_{\mathfrak{B}}\right)$ is a morphism of $L$-Top.

Proof. Let $b \in \tau_{\mathfrak{B}}$. Then $b=\bigvee\left\{c \in L^{Y} \mid \operatorname{st}(c, \mathcal{B}) \leqslant b\right.$ for some $\left.\mathcal{B} \in \mathfrak{B}\right\}$. Let us show that $f^{\leftarrow}(b) \in \tau_{\mathfrak{l}}$, by proving that

$$
\begin{aligned}
& f^{\leftarrow}(b)=\bigvee\left\{f^{\leftarrow}(c) \mid c \in L^{Y}, \operatorname{st}(c, \mathcal{B}) \leqslant b \text { for some } \mathcal{B} \in \mathfrak{B}\right\} \leqslant \bigvee\left\{f^{\leftarrow}(c) \mid c \in L^{Y}, f^{\leftarrow}(\operatorname{st}(c, \mathcal{B}))\right. \\
& \left.\leqslant f^{\leftarrow}(b) \text { for some } \mathcal{B} \in \mathfrak{B}\right\} \leqslant \bigvee\left\{a \in L^{X} \mid \operatorname{st}\left(a, f^{-1}(\mathcal{B})\right)\right. \\
& \left.\leqslant f^{\leftarrow}(b) \text { for some } f^{-1}(\mathcal{B}) \in \mathfrak{U}\right\} \leqslant \operatorname{int}\left(f^{\leftarrow}(b)\right) .
\end{aligned}
$$

The first equality follows from Property 2.1(1) and the inequalities follow from Properties 2.1 and Proposition 3.2(6).

Thus, the correspondence

$$
(X, \mathfrak{U}) \in L \text {-Unif } \longmapsto\left(X, \tau_{\mathfrak{U}}\right) \in L \text {-Top }
$$

is functorial and we have a functor $F_{3}: L$-Unif $\rightarrow L$-Top such that the diagram

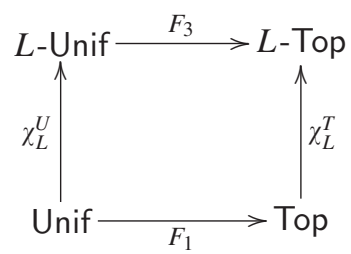

commutes (where $\chi_{L}^{U}$ denotes the uniform version of the embedding functor $\chi_{L}^{T}$ : Top $\rightarrow L$-Top). This shows that, for frames $L$, the notion of a covering $L$-valued uniform space relates to a uniform space in a way similar to that in which an $L$-valued topological space is related to a topological space.

\section{Residuated $L$-valued uniform spaces}

The category of residuated $L$-valued uniform spaces that we introduce in this section is based on the notion of residuated pairs. It has nice features: it is equivalent to the category of covering $L$-valued uniform spaces on one hand and also, for a large class of lattices (more precisely, Girard quantales), captures a significant class of Hutton uniformities.

Originally, Galois connections were expressed in a contravariant form with transformations that reverse order [3]. A Galois connection between partially ordered sets $A$ and $B$ is a pair $(f, g)$ of order-reversing maps $f: A \rightarrow B$ and $g: B \rightarrow A$ such that

$$
\operatorname{id}_{A} \leqslant g \cdot f, \quad \operatorname{id}_{B} \leqslant f \cdot g \text { equivalently, } \quad b \leqslant f(a) \text { iff } a \leqslant g(b) .
$$

We denote by $\operatorname{Gal}(A, B)$ the set of all order-reversing maps $f: A \rightarrow B$ for which there exists $f^{+}: B \rightarrow A$ such that $\left(f, f^{+}\right)$is a Galois connection.

Nowadays many authors prefer to work with Galois connections in the covariant form by its convenience (the survey [5] contains a list of references to this form). We refer to this dualized form as a residuated pair: a residuated pair between the partially ordered sets $A$ and $B$ is a pair $(f, g)$ of order-preserving maps $f: A \rightarrow B$ and $g: B \rightarrow A$ such that

$$
f \cdot g \leqslant \operatorname{id}_{B}, \quad \operatorname{id}_{A} \leqslant g \cdot f \text { equivalently, } \quad f(a) \leqslant b \text { iff } a \leqslant g(b) .
$$


The map $f: A \rightarrow B$ in a residuated pair $(f, g)$ is called residuated and the map $g: B \rightarrow A$ is called residual. Thus, $f$ is residuated (resp. residual) if and only if $f \in \operatorname{Gal}\left(A, B^{\mathrm{op}}\right.$ ) (resp. $f \in \operatorname{Gal}\left(A^{\mathrm{op}}, B\right)$ ). If $A$ and $B$ are complete lattices then $f: A \rightarrow B$ is residuated if and only if it is join-preserving. For any sets $X$ and $Y$ and any map $f: X \rightarrow Y$, $\left(f^{\leftarrow}, f^{\rightarrow}\right)$ is a residuated pair (see [30]).

Now consider the image and preimage operators $f \Rightarrow:\left(L^{X}\right)^{L^{X}} \rightarrow\left(L^{Y}\right)^{L^{Y}}$ and $f \Leftarrow:\left(L^{Y}\right)^{L^{Y}} \rightarrow\left(L^{X}\right)^{L^{X}}$, defined by $f^{\Rightarrow}(\varphi)=f^{\rightarrow} \cdot \varphi \cdot f^{\leftarrow}$ and $f^{\leftarrow}(\psi)=f^{\leftarrow} \cdot \psi \cdot f^{\rightarrow}$ :
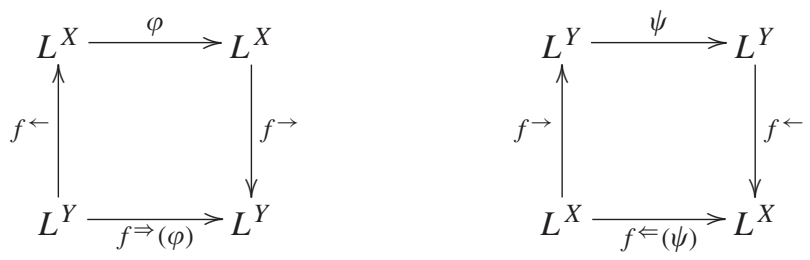

Again, $(f \Leftarrow, f \Rightarrow)$ is a residuated pair.

Let $\mathcal{H}(L, X)$ denote the collection of all join-preserving mappings $\phi: L^{X} \rightarrow L^{X}$ (in particular, $\phi\left(1_{\varnothing}\right)=1_{\varnothing}$ ), partially ordered by

$$
\phi_{1} \leqslant \phi_{2} \equiv \phi_{1}(a) \leqslant \phi_{2}(a) \text { for every } a \in L^{X} .
$$

Let $\phi \in \mathcal{H}(L, X)$. We say that $a \in L^{X}$ is $\phi$-small if

$$
a * b \neq 1_{\varnothing} \Rightarrow a \leqslant \phi(b) \text { for any } b \in L^{X} .
$$

Further, we say that $\phi$ is:

(1) symmetric whenever $\phi(a) * b=1_{\emptyset}$ iff $a * \phi(b)=1_{\emptyset}$ (or, equivalently, whenever $b \leqslant \phi(a) \rightarrow 1_{\emptyset}$ iff $\phi(b) \leqslant a \rightarrow 1_{\emptyset}$ ), for arbitrary $a, b \in L^{X}$

(2) an L-entourage of $X$ if $\left\{a \in L^{X} \mid a\right.$ is $\phi$-small $\}$ is an $L$-cover of $X$.

Proposition 4.1. Let $\phi \in \mathcal{H}(L, X)$ :

(1) If $\phi$ is symmetric then, for any $a, b \in L^{X}$,

$$
b \rightarrow 1_{\emptyset} \leqslant \phi(a) \rightarrow 1_{\emptyset} \Longleftrightarrow \phi\left(b \rightarrow 1_{\emptyset}\right) \leqslant a \rightarrow 1_{\emptyset} .
$$

(2) If $L$ is a Girard quantale then the converse to (1) holds. In that case, $\phi$ is symmetric if and only if

$$
\phi(a) \leqslant b \Longleftrightarrow \phi\left(b \rightarrow 1_{\emptyset}\right) \leqslant a \rightarrow 1_{\emptyset} .
$$

(3) If $\phi$ is an L-entourage then $\phi(a) \geqslant a$ for every $a \in L^{X}$.

(4) If $L$ is atomic (i.e. every element of $L$ is the join of all atoms below it) then the converse to (3) holds.

Proof. (1) and (2) These are obvious.

(3) For each $a \in L^{X}$,

$$
\begin{aligned}
a=1_{X} * a & =\left(\bigvee\left\{b \in L^{X} \mid b \text { is } \phi \text {-small }\right\}\right) * a=\bigvee\left\{b * a \mid b * a \neq 1_{\emptyset}, b \text { is } \phi \text {-small }\right\} \\
& \leqslant \bigvee\left\{b \mid b * a \neq 1_{\varnothing}, b \text { is } \phi \text {-small }\right\} \leqslant \phi(a) .
\end{aligned}
$$

(4) Let $\phi: L^{X} \rightarrow L^{X}$ be such that $\phi(a) \geqslant a$ for every $a \in L^{X}$. For each atom $\alpha$ of $L$, it follows that $\alpha \wedge 1_{\{x\}} \in L^{X}$ is an atom in $L^{X}$. Then $1_{\emptyset} \neq\left(\alpha \wedge 1_{\{x\}}\right) * b \leqslant \alpha \wedge 1_{\{x\}} \wedge b \leqslant \alpha \wedge 1_{\{x\}}$ implies $\alpha \wedge 1_{\{x\}} \leqslant b$. In particular, this means that every $\alpha \wedge 1_{\{x\}}$ is $\phi$-small. Hence, for every $x \in X$,

$$
\begin{aligned}
\left(\bigvee\left\{a \in L^{X} \mid a \text { is } \phi \text {-small }\right\}\right)(x) & \geqslant\left(\bigvee\left\{\alpha \wedge 1_{\{x\}} \mid \alpha \text { is an atom of } L\right\}\right)(x) \\
& =\bigvee\{\alpha \mid \alpha \text { is an atom of } L\}=1
\end{aligned}
$$


Remark 4.2. If $L$ fails to be atomic then the converse to Proposition 4.1(3) is not true in general. The unit interval, with its usual order, gives rise to a couple of examples, when it is endowed with the following two well-known quantale structures (in fact a similar statement holds for any quantale structure defined on $[0,1]$ ):

(1) $([0,1], \leqslant, \wedge)$, (the unit interval considered as a Heyting algebra).

(2) $\left([0,1], \leqslant, T_{m}\right)$ (the unit interval considered as an $M V$-algebra), where $T_{m}(\alpha, \beta)=\max \{\alpha+\beta-1,0\}$ for each $\alpha, \beta \in[0,1]$ is the Luckasiewicz $T$-norm.

Indeed, the identity map $\operatorname{id}_{X}: L^{X} \rightarrow L^{X}$ belongs to $\mathcal{H}(L, X)$ and it is not difficult to check that, in the first case, $a \in L^{X}$ is id $\operatorname{~}_{X}$-small iff $a=1_{\varnothing}$, while in the second $a \in L^{X}$ is $\operatorname{id}_{X}$-small iff $a=\alpha \wedge 1_{x}$ for some $\alpha \leqslant \frac{1}{2}$ and $x \in X$. So, either $\bigvee\left\{a \in L^{X} \mid a\right.$ is $\operatorname{id}_{X}$-small $\}=1_{\emptyset}$ or $\bigvee\left\{a \in L^{X} \mid a\right.$ is $\operatorname{id}_{X}$-small $\}=\frac{1}{2}$; in both cases id $X$ fails to be an $L$-entourage.

Definition 4.3. We say that a pair $(X, \mathcal{D})$ consisting of a set $X$ and a nonempty subset $\mathcal{D}$ of $\mathcal{H}(L, X)$ is a residuated $L$-uniform space whenever the following conditions hold:

(R1) Each $\phi \in \mathcal{D}$ is an $L$-entourage of $X$.

(R2) $\phi_{1} \in \mathcal{D}$ and $\phi_{1} \leqslant \phi_{2} \in \mathcal{H}(L, X)$ implies $\phi_{2} \in \mathcal{D}$.

(R3) For each $\phi_{1}, \phi_{2} \in \mathcal{D}$ there exists $\phi_{3} \in \mathcal{D}$ such that $\phi_{3} \leqslant \phi_{1}$ and $\phi_{3} \leqslant \phi_{2}$.

(R4) For each $\phi_{1} \in \mathcal{D}$ there exists $\phi_{2} \in \mathcal{D}$ such that $\phi_{2} \cdot \phi_{2} \leqslant \phi_{1}$.

(R5) For each $\phi_{1} \in \mathcal{D}$ there exists a symmetric $\phi_{2} \in \mathcal{D}$ such that $\phi_{2} \leqslant \phi_{1}$.

A base for the uniformity $\mathcal{D}$ is any subcollection of $\mathcal{D}$ from which $\mathcal{D}$ can be recovered by applying condition (R2). Note that (R5) means that $\mathcal{D}$ has a base of symmetric entourages.

The morphisms (uniform morphisms) of the resulting category $L$-Unif ${ }^{r}$ are the maps $f:(X, \mathcal{D}) \rightarrow(Y, \mathcal{E})$ satisfying $f^{\Leftarrow}(\phi) \in \mathcal{D}$ for every $\phi \in \mathcal{E}$.

When $L=2$, these are precisely the classical uniformities, described in terms of axialities [5] (cf. also [6, Section 5]). Since the symmetry condition (R5) is now explicitly axiomatized, by dropping it we have the notion of an (residuated) $L$-quasi-uniform space. When $L=2$ this gives precisely the classical notion of a quasi-uniform space.

It is now our purpose to prove that the categories $L$-Unif ${ }^{r}$ and $L$-Unif are isomorphic.

Let $\mathfrak{U}$ be a covering $L$-uniformity on $X$ and, for each $\mathcal{A} \in \mathfrak{U}$, let

$$
\begin{aligned}
& \phi_{\mathcal{A}}: L^{X} \rightarrow L^{X} \\
& a \mapsto \operatorname{st}(a, \mathcal{A}) .
\end{aligned}
$$

The next proposition with the exception of symmetry follows immediately from Proposition 3.2 and lists some basic properties of these maps.

\section{Proposition 4.4.}

(1) For each $\mathcal{A} \in \mathcal{U}, \phi_{\mathcal{A}} \in \mathcal{H}(L, X)$. Also, since $\operatorname{st}(\mathcal{A}) \in \mathcal{U}, \phi_{\mathrm{st}(\mathcal{A})} \in \mathcal{H}(L, X)$ and $\mathcal{A} \preccurlyeq \operatorname{st}(\mathcal{A})$.

(2) If $\mathcal{A} \preccurlyeq \mathcal{B}$ then $\phi_{\mathcal{A}} \leqslant \phi_{\mathcal{B}}$.

(3) For every $a \in L^{X}, a \leqslant \phi_{\mathcal{A}}(a)$.

(4) Each $a \in \mathcal{A}$ is $\phi_{\mathcal{A}^{-} \text {-small. }}$

(5) Each $\phi_{\mathcal{A}}$ is a symmetric L-entourage.

(6) If $\operatorname{st}(\mathcal{A}) \preccurlyeq \mathcal{B}$ then $\phi_{\mathcal{A}} \cdot \phi_{\mathcal{A}} \leqslant \phi_{\mathcal{B}}$.

Proof. (5) It follows from the following equivalence:

$$
\phi_{\mathcal{A}}(a) * b \neq 1_{\varnothing} \Leftrightarrow \exists c \in \mathcal{A}: c * a \neq 1_{\varnothing} \quad \text { and } \quad c * b \neq 1_{\varnothing} \Leftrightarrow a * \phi_{\mathcal{A}}(b) \neq 1_{\emptyset} .
$$

The next corollary is an immediate consequence of Proposition 4.4.

Corollary 4.5. Let $\mathfrak{U}$ be a covering L-uniformity on $X$. Then $\left\{\phi_{\mathcal{A}} \mid \mathcal{A} \in \mathfrak{U}\right\}$ is a base for a residuated uniformity $\mathcal{D}_{\mathfrak{U}}$ on $X$. 
Proposition 4.6. For every uniform homomorphism $f:(X, \mathfrak{U}) \rightarrow(Y, \mathfrak{B})$, the map $f:\left(X, \mathcal{D}_{\mathfrak{U}}\right) \rightarrow\left(Y, \mathcal{D}_{\mathfrak{B}}\right)$ is uniform.

Proof. We need to prove that $f^{\leftarrow}(\phi)=f^{\leftarrow \cdot \phi} \cdot f^{\rightarrow} \in \mathcal{D}_{\mathfrak{U}}$ for every $\phi \in \mathcal{D}_{\mathfrak{B}}$. So, let $\mathcal{B} \in \mathfrak{B}$ such that $\phi_{\mathcal{B}} \leqslant \phi$ and $\mathcal{A} \in \mathfrak{U}$ satisfying $\mathcal{A} \preccurlyeq f^{-1}[\mathcal{B}]$. Combining results in Proposition 3.2(3) and (6), and Proposition 4.4(2), for every $a \in L^{X}$ we may write:

$$
\begin{aligned}
\phi_{\mathcal{A}}(a) & \leqslant \phi_{f^{-1}[\mathcal{B}]}(a)=\operatorname{st}\left(a, f^{-1}[\mathcal{B}]\right) \leqslant \operatorname{st}\left(f^{\leftarrow}\left(f^{\rightarrow}(a)\right), f^{-1}[\mathcal{B}]\right) \leqslant f^{\leftarrow}\left(\operatorname{st}\left(f^{\rightarrow}(a), \mathcal{B}\right)\right) \\
& =f^{\leftarrow}\left(\phi_{\mathcal{B}}\left(f^{\rightarrow}(a)\right)\right) \leqslant f^{\leftarrow}\left(\phi\left(f^{\rightarrow}(a)\right)\right)=\left(f^{\leftarrow} \cdot \phi \cdot f^{\rightarrow}\right)(a) .
\end{aligned}
$$

Finally, we have:

Corollary 4.7. The correspondences $(X, \mathfrak{U}) \mapsto\left(X, \mathcal{D}_{\mathfrak{U}}\right)$ and $f \mapsto f$ given by Corollary 4.5 and Proposition 4.6 establish a functor $\Phi: L$-Unif $\rightarrow L$-Unif ${ }^{r}$.

Conversely, let $\mathcal{D}$ be a residuated $L$-uniformity on $X$. For each $\phi \in \mathcal{D}$, consider the $L$-cover

$$
\mathcal{A}_{\phi}=\left\{a \in L^{X} \mid a \text { is } \phi \text {-small }\right\} .
$$

The following proposition lists some of the basic properties of these covers.

\section{Proposition 4.8.}

(1) If $\phi_{1} \leqslant \phi_{2}$ then $\mathcal{A}_{\phi_{1}} \preccurlyeq \mathcal{A}_{\phi_{2}}$.

(2) $\mathcal{A}_{\phi_{1} \wedge \phi_{2}} \preccurlyeq \mathcal{A}_{\phi_{1}} \wedge \mathcal{A}_{\phi_{2}}$.

(3) If $\phi_{1} \cdot \phi_{1} \cdot \phi_{1} \leqslant \phi_{2}$ and $\phi_{1}$ is symmetric then $\operatorname{st}\left(\mathcal{A}_{\phi_{1}}\right) \preccurlyeq \mathcal{A}_{\phi_{2}}$.

Proof. (1) It is obvious since any $\phi_{1}$-small element is $\phi_{2}$-small whenever $\phi_{1} \leqslant \phi_{2}$.

(2) It is an immediate consequence of the previous property.

(3) Let $\operatorname{st}\left(a, \mathcal{A}_{\phi_{1}}\right) \in \operatorname{st}\left(\mathcal{A}_{\phi_{1}}\right)$. Then $\operatorname{st}\left(a, \mathcal{A}_{\phi_{1}}\right)=\bigvee\left\{b \in \mathcal{A}_{\phi_{1}} \mid b * a \neq 1_{\emptyset}\right\} \leqslant \phi_{1}(a)$ (because each such $b$ is $\phi_{1}$-small). It suffices now to check that $\phi_{1}(a)$ is $\phi_{2}$-small. By the symmetry of $\phi_{1}, \phi_{1}(a) * b \neq 1_{\varnothing}$ if and only if $a * \phi_{1}(b) \neq 1_{\emptyset}$, so $\phi_{1}(a) * b \neq 1_{\emptyset}$ implies $a \leqslant\left(\phi_{1} \cdot \phi_{1}\right)(b)$, and consequently, $\phi_{1}(a) \leqslant \phi_{1}^{3}(b) \leqslant \phi_{2}(b)$, which shows that $\phi_{1}(a)$ is indeed $\phi_{2}$-small.

From this result it follows immediately that:

Corollary 4.9. Let $\mathcal{D}$ be a residuated L-uniformity on $X$. Then $\left\{\mathcal{A}_{\phi} \mid \phi \in \mathcal{D}\right\}$ is a base for a covering L-uniformity $\mathfrak{U}_{\mathcal{D}}$ on $X$.

Lemma 4.10. Let $f: X \rightarrow Y, a \in L^{X}$ and $\psi \in\left(L^{Y}\right)^{L^{Y}}$. If a is $f^{\Leftarrow}(\psi)$-small then $f^{\rightarrow}(a)$ is $\psi$-small.

Proof. Let $f^{\rightarrow}(a) * b \neq 1_{\emptyset}$ equivalently (cf. Lemma 2.1(8)) $a * f^{\leftarrow}(b) \neq 1 \emptyset$, which implies

$$
a \leqslant f^{\leftarrow}(\psi)\left(f^{\leftarrow}(b)\right)=\left(f^{\leftarrow} \cdot \psi \cdot f^{\rightarrow} \cdot f^{\leftarrow}\right)(b) \leqslant\left(f^{\leftarrow} \cdot \psi\right)(b),
$$

since $f^{\rightarrow} \cdot f^{\leftarrow} \leqslant \operatorname{id}_{L^{Y}}$. Thus, $a \leqslant\left(f^{\leftarrow} \cdot \psi\right)(b)$ and, consequently, $f^{\rightarrow}(a) \leqslant\left(f^{\rightarrow} \cdot f^{\leftarrow} \cdot \psi\right)(b) \leqslant \psi(b)$.

Proposition 4.11. For every uniform homomorphism $f:(X, \mathcal{D}) \rightarrow(Y, \mathcal{E})$, the map $f:\left(X, \mathfrak{U}_{\mathcal{D}}\right) \rightarrow\left(Y, \mathfrak{U}_{\mathcal{E}}\right)$ is uniform.

Proof. For each $\mathcal{A} \in \mathfrak{U}_{\mathcal{E}}$ let $\psi \in \mathcal{E}$ such that $\mathcal{A}_{\psi} \preccurlyeq \mathcal{A}$. We need to prove that there exists $\phi \in \mathcal{D}$ for which $\mathcal{A}_{\phi} \preccurlyeq f^{-1}[\mathcal{A}]$. Since, by hypothesis, for each such $\psi$ there exists $\phi \in \mathcal{D}$ satisfying $\phi \leqslant f \Leftarrow(\psi)$, which implies $\mathcal{A}_{\phi} \preccurlyeq \mathcal{A}_{f} \Leftarrow(\psi)$, it suffices to show that $\mathcal{A}_{f} \Leftarrow(\psi) \preccurlyeq f^{-1}[\mathcal{A}]$. So let $b \in L^{X}$ be $f^{\Leftarrow}(\psi)$-small. Then $f^{\rightarrow}(b)$ is $\psi$-small by Lemma 4.10. But $\mathcal{A}_{\psi} \preccurlyeq \mathcal{A}$. Consequently, there exists $a \in \mathcal{A}$ such that $f^{\rightarrow}(b) \leqslant a$. Hence $b \leqslant f^{\leftarrow}(a)$. 
Corollary 4.12. The correspondences $(X, \mathcal{D}) \mapsto\left(X, \mathfrak{U}_{\mathcal{D}}\right)$ and $f \mapsto f$ given by Corollary 4.9 and Proposition 4.11 define a functor $\Psi: L$-Unif ${ }^{r} \rightarrow L$-Unif.

Now let us show that $\Phi \cdot \Psi=\mathrm{id}_{L-\text { Unif }^{r}}$ and $\Psi \cdot \Phi=\mathrm{id}_{L-\text { Unif. }}$

Lemma 4.13. For any $L$-covers $\mathcal{A}$ and $\mathcal{B}$ of $X$ and any $\phi, \psi \in \mathcal{H}(L, X)$ we have:

(1) $\mathcal{A} \preccurlyeq \mathcal{A}_{\phi_{\mathcal{A}}}$.

(2) If $\operatorname{st}(\mathcal{A}) \preccurlyeq \mathcal{B}$ then $\mathcal{A}_{\phi_{\mathcal{A}}} \preccurlyeq \mathcal{B}$.

(3) $\phi_{\mathcal{A}_{\phi}} \leqslant \phi$.

(4) If $\phi \cdot \phi \leqslant \psi$ then $\phi \leqslant \phi_{\mathcal{A}_{\psi}}$.

Proof. (1) For every $a \in \mathcal{A}, a * b \neq 1_{\emptyset}$ implies $a \leqslant \operatorname{st}(b, \mathcal{A})=\phi_{\mathcal{A}}(b)$, so $a$ is $\phi_{\mathcal{A}}$-small.

(2) Let $a \in L^{X}$ be $\phi_{\mathcal{A}}$-small. We need to show that $a \leqslant b$ for some $b \in \mathcal{B}$. The case $a=1_{\emptyset}$ is trivial. If $a \neq 1_{\emptyset}$ then, since $a=a * \bigvee_{c \in \mathcal{A}} c$, there is some $c \in \mathcal{A}$ for which $a * c \neq 1_{\emptyset}$. Then $a \leqslant \phi_{\mathcal{A}}(c)=\operatorname{st}(c, \mathcal{A}) \leqslant b$ for some $b \in \mathcal{B}$.

(3) $\phi_{\mathcal{A}_{\phi}}(a)=\operatorname{st}\left(a, \mathcal{A}_{\phi}\right)=\bigvee\left\{b \in L^{X} \mid b\right.$ is $\phi$-small, $\left.b * a \neq 1_{\emptyset}\right\} \leqslant \phi(a)$.

(4) If $\phi \cdot \phi \leqslant \psi$ then

$$
\begin{aligned}
\phi(a) & =\phi(a) * \bigvee\left\{b \in L^{X} \mid b \text { is } \phi \text {-small }\right\}=\bigvee\left\{\phi(a) * b \mid b \text { is } \phi \text {-small, } \phi(a) * b \neq 1_{\varnothing}\right\} \\
& \leqslant(\phi \cdot \phi)(a) \leqslant \psi(a) .
\end{aligned}
$$

Theorem 4.14. The functors $\Phi$ and $\Psi$ establish an isomorphism between the categories $L$-Unif and L-Unif ${ }^{r}$.

Proof. Properties (1) and (2) of Lemma 4.13 imply immediately that, for any covering uniformity $\mathfrak{U}, \mathfrak{U}_{\mathcal{D}_{\mathfrak{l}}}=\mathfrak{U}$. Similarly, properties (3) and (4) of Lemma 4.13 ensure us that, for any residuated uniformity $\mathcal{D}, \mathcal{D}_{\mathfrak{U}_{\mathcal{D}}}=\mathcal{D}$. Hence $\Phi \cdot \Psi=\mathrm{id}_{L-U n i f}{ }^{r}$ and $\Psi \cdot \Phi=\mathrm{id}_{L-\text { Unif. }}$

\section{The relationship with Hutton uniformities}

Let $L$ be a Girard quantale. Each $\phi \in \mathcal{H}(L, X)$, being join-preserving, has a right adjoint $\phi^{*}: L^{X} \rightarrow L^{X}$. Let $\phi^{-1}: L^{X} \rightarrow L^{X}$ be defined by

$$
\phi^{-1}(a)=\phi^{*}\left(a \rightarrow 1_{\emptyset}\right) \rightarrow 1_{\emptyset}=\bigwedge\left\{b \rightarrow 1_{\emptyset} \in L^{X} \mid \phi(b) \leqslant a \rightarrow 1_{\emptyset}\right\}
$$

It is easy to check that $\left(\phi^{-1}\right)^{-1}=\phi$ and $\phi_{1} \leqslant \phi_{2} \Leftrightarrow \phi_{2}^{*} \leqslant \phi_{1}^{*} \Leftrightarrow \phi_{1}^{-1} \leqslant \phi_{2}^{-1}$.

In the original definition of Hutton [15], the involved lattice is a completely distributive one with an order reversing involution $\left(L, \leqslant,^{\prime}\right)$. Our context is a bit more general and complete distributivity is not needed (cf. [32]). We say that a nonempty subset $\mathcal{D}$ of $\mathcal{H}(L, X)$ is a Hutton $L$-uniformity on $X$ if $\mathcal{D}$ satisfies the following axioms:

(H1) $\forall \phi \in \mathcal{D}, \phi(a) \geqslant a$ for all $a \in L^{X}$.

(H2) $\phi_{1} \in \mathcal{D}$ and $\phi_{1} \leqslant \phi_{2} \in \mathcal{H}(L, X)$ implies $\phi_{2} \in \mathcal{D}$.

(H3) For each $\phi_{1}, \phi_{2} \in \mathcal{D}$ there exists $\phi_{3} \in \mathcal{D}$ such that $\phi_{3} \leqslant \phi_{1}$ and $\phi_{3} \leqslant \phi_{2}$.

(H4) For each $\phi_{1} \in \mathcal{D}$ there exists $\phi_{2} \in \mathcal{D}$ such that $\phi_{2} \cdot \phi_{2} \leqslant \phi_{1}$.

(H5) $\forall \phi \in \mathcal{D}, \phi^{-1} \in \mathcal{D}$.

According to Hutton [15] an element $\phi \in \mathcal{D}$ is symmetric whenever $\phi=\phi^{-1}$. The following lemma shows that our definition of symmetry coincides with the original one of Hutton.

Lemma 5.1. Let $L$ be a Girard quantale. For each $\phi \in \mathcal{H}(L, X), \phi$ is symmetric if and only if $\phi=\phi^{-1}$.

Proof. If $\phi$ is symmetric then $\phi(b) \leqslant a \rightarrow 1_{\emptyset} \Leftrightarrow \phi(a) \leqslant b \rightarrow 1_{\emptyset}$, which implies

$$
\phi^{-1}(a)=\bigwedge\left\{b \rightarrow 1_{\emptyset} \in L^{X} \mid \phi(b) \leqslant a \rightarrow 1_{\emptyset}\right\}=\bigwedge\left\{b \rightarrow 1_{\emptyset} \in L^{X} \mid \phi(a) \leqslant b \rightarrow 1_{\emptyset}\right\}=\phi(a) .
$$


Conversely, if $\phi=\phi^{-1}$ then

$$
\begin{aligned}
\phi(a) * b=1_{\emptyset} & \Leftrightarrow \phi^{-1}(a) * b=1_{\varnothing} \Leftrightarrow b \leqslant \phi^{-1}(a) \rightarrow 1_{\emptyset}=\phi^{*}\left(a \rightarrow 1_{\emptyset}\right) \\
& \Leftrightarrow \phi(b) \leqslant a \rightarrow 1_{\emptyset} \Leftrightarrow a * \phi(b)=1_{\varnothing},
\end{aligned}
$$

which shows that $\phi$ is symmetric.

Lemma 5.2. For each $\phi \in \mathcal{H}(L, X), \phi^{-1} \cdot \phi$ and $\phi \cdot \phi^{-1}$ are symmetric.

Proof. We only prove the first assertion (the other may be proved similarly):

$$
\begin{aligned}
\phi^{-1}(\phi(a)) * b=1_{\emptyset} & \Leftrightarrow b \leqslant \phi^{-1}(\phi(a)) \rightarrow 1_{\varnothing}=\phi^{*}\left(\phi(a) \rightarrow 1_{\emptyset}\right) \Leftrightarrow \phi(b) \leqslant \phi(a) \rightarrow 1_{\emptyset} \\
& \Leftrightarrow \phi(a) * \phi(b)=1_{\emptyset} \Leftrightarrow \phi(a) \leqslant \phi(b) \rightarrow 1_{\emptyset} \Leftrightarrow a \leqslant \phi^{*}\left(\phi(b) \rightarrow 1_{\emptyset}\right)=\phi^{-1}(\phi(b)) \rightarrow 1_{\emptyset} \\
& \Leftrightarrow \phi^{-1}(\phi(b)) * a=1_{\emptyset} \Leftrightarrow a * \phi^{-1}(\phi(b))=1_{\emptyset} .
\end{aligned}
$$

Proposition 5.3. Let $L$ be a Girard quantale and $\mathcal{D} \subseteq \mathcal{H}(L, X)$. Then $\mathcal{D}$ satisfies $(\mathrm{H} 2),(\mathrm{H} 3),(\mathrm{H} 4)$, and $(\mathrm{H} 5)$ if and only if it satisfies (R2), (R3), (R4), and (R5).

Proof. Axioms (H2), (H3), and (H4) are precisely (R2), (R3), and (R4), respectively. Finally, in the presence of these axioms, (H5) $\Leftrightarrow(\mathrm{R} 5): \Rightarrow$ Let $\phi \in \mathcal{D}$ and use (H4) to get $\psi \in \mathcal{D}$ such that $\psi \cdot \psi \leqslant \phi$. By (H5), $\psi^{-1} \in \mathcal{D}$. Applying (H3), consider $\xi \in \mathcal{D}$ such that $\xi \leqslant \psi$ and $\xi \leqslant \psi^{-1}$. Then $\xi^{-1} \leqslant\left(\psi^{-1}\right)^{-1}=\psi$ and $\xi \cdot \xi^{-1} \leqslant \psi \cdot \psi \leqslant \phi$. Since $\xi \cdot \xi^{-1}$ is symmetric by Lemma 5.2, $\mathcal{D}$ satisfies (R5).

$\Leftarrow$ Let $\phi \in \mathcal{D}$. By (R5) there exists a symmetric $\psi \in \mathcal{D}$ such that $\psi \leqslant \phi$, that is, $\psi^{-1} \leqslant \phi^{-1}$. By Lemma 5.1, $\psi^{-1}=\psi \in \mathcal{D}$, thus $\phi^{-1} \in \mathcal{D}$ by (R2).

Therefore, since axiom (H1) is a consequence of (R1) by Proposition 4.1(3), we have immediately:

Corollary 5.4. Let L be a Girard quantale. Every residuated L-uniformity on X is a Hutton uniformity on X.

The converse is not true in general (recall Proposition 4.1(4)).

Remark 5.5. In the classical context, for a set $X$, binary relations $R \subseteq X \times X$ are described by Galois connections between power sets in two particularly simple ways [5]:

(1) A Galois connection between $\mathcal{P}(X)$ and $\mathcal{P}(X)$ is called a polarity [3] on $\mathcal{P}(X)$. Any relation $R \subseteq X \times X$ induces a polarity $\left(R_{\forall}, R^{\forall}\right)$ on $\mathcal{P}(X)$, defined by

$$
\begin{array}{ll}
R_{\forall}(A):=\{y \in X \mid \forall x \in A(x, y) \in R\} & \text { for } A \subseteq X, \\
R^{\forall}(B):=\{x \in X \mid \forall y \in B(x, y) \in R\} & \text { for } B \subseteq X .
\end{array}
$$

Conversely, every polarity $(f, g)$ on $\mathcal{P}(X)$ induces a relation

$$
\left.(x, y) \in R_{(f, g)} \equiv y \in f(\{x\}) \quad \text { (or equivalently, } x \in g(\{y\})\right) .
$$

Since $\left(\left(R_{(f, g)}\right)_{\forall},\left(R_{(f, g)}\right)^{\forall}\right)=(f, g)$ and $R_{\left(R_{\forall}, R^{\forall}\right)}=R$, there is a bijection between relations $R \subseteq X \times X$ and polarities on $\mathcal{P}(X)$.

(2) The covariant case of Galois connections between power sets also describe all binary relations $R \subseteq X \times X$. Indeed, any relation $R \subseteq X \times X$ induces an axiality [5] $\left(R_{\exists}, R^{\forall}\right)$ on $\mathcal{P}(X)$, that is, a Galois connection between $\mathcal{P}(X)$ and $\mathcal{P}(X)^{\mathrm{op}}$, defined by

$$
\begin{aligned}
& R_{\exists}(A):=\{y \in X \mid \exists x \in A(x, y) \in R\} \quad \text { for } A \subseteq X, \\
& \left.R^{\forall}(B):=\{x \in X \mid \forall y \in X(x, y) \in R \Rightarrow y \in B)\right\} \text { for } B \subseteq X .
\end{aligned}
$$

If we define, for each axiality $(f, g)$ on $\mathcal{P}(X)$, the relation

$$
(x, y) \in R_{(f, g)} \equiv y \in f(\{x\}),
$$

then $\left(\left(R_{(f, g)}\right)_{\exists},\left(R_{(f, g)}\right)^{\forall}\right)=(f, g)$ and $R_{\left(R_{\exists}, R^{\forall}\right)}=R$. 
Under bijection (1) (resp. (2)), entourages $E$, i.e. reflexive relations, correspond to polarities (resp. axialities) that are expansive on atoms, that is, $\{x\} \subseteq E_{\forall}(\{x\})$ (resp. $\{x\} \subseteq E_{\exists}(\{x\})$ ) for every $x \in X$. Nevertheless, to be expansive on atoms, has a different meaning in polarities and axialities; in the case of polarities, this is equivalent to $\{A \subseteq X \mid A \subseteq$ $\left.E_{\forall}(A)\right\}$ being a cover of $X$ while in the case of axialities, it is equivalent to $E_{\exists}$ being expansive on subsets (cf. axiom (H1)), and also to the collection of all $E_{\exists}$-small sets being a cover of $X$ (cf. axiom (R1)).

This equivalence, in the case of axialities, relies on two facts: firstly, $\mathcal{P}(X)$ being atomic, its elements can be written as unions of points (atoms) and secondly, the involved maps $E_{\exists}$ are sup-preserving.

If the lattice 2 is replaced by a general $L$, even if the previous situation models a relation between polarities (axialites) from $L^{X}$ to $L^{X}$ and $L$-valued binary relations, i.e:, elements in $L^{X \times X}$ (see [11]), one cannot expect that, in this general case, either working with expansive maps or working with maps whose small elements form a cover might be still equivalent. And it is really the case, as examples in Remark 4.2 show. Indeed, the equivalence established between $L$-valued binary relations and some family of sup-preserving maps from $L^{X}$ to $L^{X}$, (see [11]) transforms reflexive $L$-valued binary relations (maps $f$ from $X \times X \rightarrow L$, such that $f(x, x)=1$ ) into expansive maps.

It would be interesting to investigate under which conditions the work in [6] (Sections 3 and 4; see also [22]) may be extended to our setting here, showing whether uniform structures stated in terms of residuated maps $L^{X} \rightarrow L^{X}$ (that is, elements of $\mathcal{H}(L, X)$ ) as we defined in Section 4, may be equivalently described in terms of Galois maps $L^{X} \rightarrow L^{X}$ (that is, residuated maps $L^{X} \rightarrow\left(L^{X}\right)^{\text {op }}$ ).

\section{6. $L$-valued frames}

For the motivation and justification for this notion see [26]. There the authors show that levels and level topologies may be interpreted as a system of frame homomorphisms satisfying some categorical conditions. Indeed, $L$-fuzzy and traditional structures, can be related via the functor $l_{L}$ and its levels $\left\{l_{\alpha}: \alpha \in L\right\}$ (see, among others [19,35-37,18] for topologies and $[20,24,9,10]$ for filters and uniformities).

Two relevant facts of the level (topological) functors $\left\{l_{\alpha} \mid \alpha \in L\right\}$ are:

(1) The collection $\left\{l_{\alpha} \mid \alpha \in L\right\}$ is nondecreasing (on functions).

(2) The collection $\left\{l_{\alpha}(\tau) \mid \alpha \in L\right\}$ is a subbase for $l_{L}(\tau)$, that is: $\left\langle\bigcup_{\alpha \in L} l_{\alpha}(\tau)\right\rangle=l_{L}(\tau)$.

The categorical interpretation of these properties, led Pultr and Rodabaugh [26] to introduce the notion of an $L$-valued frame (see also $[27,28]$ for more information about this notion). The main purpose is to have a general $L$-structure which relates to frames in the way $L$-topological spaces relate to topological spaces.

Let $L_{1}=L \backslash\{1\}$. For each $\alpha \in L_{1}$ and $a \in L^{X}$, let

$$
l_{\alpha}(a)=\{x \in X \mid a(x) \nless \alpha\} .
$$

This defines the $\alpha$-level mapping $l_{\alpha}: L^{X} \rightarrow 2^{X}$. For each $L$-topological space $(X, \tau)$, the associated crisp topology $l_{L}^{T}(\tau)$ is the topology on $X$ with subbase: $\left\{l_{\alpha}(a): \alpha \in L_{1}, a \in \tau\right\}$, that is:

$$
\imath_{L}^{T}(\tau)=\left\langle\bigcup\left\{l_{\alpha}(\tau): \alpha \in L_{1},\right\}\right\rangle .
$$

The correspondence $(X, \tau) \mapsto\left(X, \imath_{L}^{T}(\tau)\right)$ defines a functor $\imath_{L}^{T}: L$-Top $\rightarrow$ Top (satisfying $\imath_{L}^{T} \cdot \chi_{L}^{T}=\operatorname{id} \mathrm{Top}_{\mathrm{T}}$ ): for each $L$-continuous map $f:\left(X, \tau_{1}\right) \rightarrow\left(Y, \tau_{2}\right), f\left(X, l_{L}^{T}\left(\tau_{1}\right)\right) \rightarrow\left(Y, l_{L}^{T}\left(\tau_{2}\right)\right)$ is continuous, since $b \cdot f \in \tau_{1}$ and $f^{-1}\left(l_{\alpha}(b)\right)=l_{\alpha}(b \cdot f)$ for every $b \in \tau_{2}$.

Recall also the (dual) adjunction between $L$-Top and Frm [13,31]: the contravariant functor $\mathcal{O}_{L}: L$-Top $\rightarrow$ Frm given by $\mathcal{O}_{L}(X, \tau)=\tau$ and $\mathcal{O}_{L}(f)(b)=b \cdot f$, has a right adjoint $\Sigma_{L}:$ Frm $\rightarrow L$-Top defined by $\Sigma_{L}(M)=(\{p:$ $M \rightarrow L \mid p \in \operatorname{Frm}\},\{\widehat{m} \mid m \in M\})$, where $\widehat{m}(p)=p(m)$ and $\Sigma_{L}(h)(p)=p \cdot h$ for every $h: M \rightarrow N$.

A family of morphisms $\left(f_{i}: A \rightarrow B_{i}\right)_{i \in I}$ in a category is said to be jointly monic (also mono-source in [1]) if $f_{i} \cdot g=f_{i} \cdot h$ for every $i \in I$ imply $g=h$. The family $\left(f_{i}: A_{i} \rightarrow B\right)_{i \in I}$ is said to be jointly epic (or an epi-sink [1]) if it satisfies the dual condition. A jointly monic family $\left(f_{i}: A \rightarrow B_{i}\right)_{i \in I}$ is jointly extremally monic if, moreover, $f_{i}=g_{i} \cdot e$, for every $i \in I$, with $e$ epimorphic (that is, $e$ is "right-cancellable" with respect to the composition), implies that $e$ is an isomorphism. Dually, a jointly epic family $\left(f_{i}: A_{i} \rightarrow B\right)_{i \in I}$ is jointly extremally epic if $f_{i}=m \cdot g_{i}$ for every $i \in I$, with $m$ monomorphic (that is, $m$ is "left-cancellable" with respect to the composition), implies that $m$ is an isomorphism. 
Let $L$ be a linearly ordered complete lattice. An $L$-valued frame (shortly, $L$-frame) [26] is a system of frame homomorphisms

$$
\mathcal{M} \equiv\left(\varphi_{\alpha}^{\mathcal{M}}: \mathcal{M}^{\mathfrak{u}} \rightarrow \mathcal{M}^{\mathfrak{l}}\right)_{\alpha \in L_{1}}
$$

satisfying the following axioms:

(1) For each nonempty $S \subseteq L_{1}, \varphi_{\bigwedge S}^{\mathcal{M}}=\bigvee_{\beta \in S} \varphi_{\beta}^{\mathcal{M}}$.

(2) $\left(\varphi_{\alpha}^{\mathcal{M}}\right)_{\alpha \in L_{1}}$ is jointly extremally epic in Frm, that is, $\mathcal{M}^{\mathrm{l}}=\left\langle\bigcup_{\alpha} \varphi_{\alpha}^{\mathcal{M}}\left[\mathcal{M}^{\mathfrak{u}}\right]\right\rangle$.

(3) $\left(\varphi_{\alpha}^{\mathcal{M}}\right)_{\alpha \in L_{1}}$ is jointly monic in Frm.

An L-frame homomorphism [26] $h: \mathcal{M} \rightarrow \mathcal{N}$ is an ordered pair of frame homomorphisms

$$
h \equiv\left(h^{\mathfrak{u}}: \mathcal{M}^{\mathfrak{u}} \rightarrow \mathcal{N}^{\mathfrak{u}}, h^{\mathfrak{l}}: \mathcal{M}^{\mathfrak{l}} \rightarrow \mathcal{N}^{\mathfrak{l}}\right)
$$

satisfying $h^{\mathbb{I}} \cdot \varphi_{\alpha}^{\mathcal{M}}=\varphi_{\alpha}^{\mathcal{N}} \cdot h^{\mathfrak{u}}$ for every $\alpha \in L_{1}$. The resulting category is denoted by $L$-Frm.

It follows immediately from (2) and (3) that in any $L$-frame homomorphism $h \equiv\left(h^{\mathfrak{u}}, h^{\mathrm{l}}\right)$, each of the frame homomorphisms $h^{\mathfrak{l}}, h^{\mathbb{l}}$ guarantees the uniqueness of the other.

If $L$ is a linearly ordered complete lattice then, in the definition of $l_{\alpha}(a)$, we may replace $\nless$ by $>$ and the mappings $l_{\alpha}: \tau \rightarrow l_{L}^{T}(\tau)\left(\alpha \in L_{1}\right)$ given by (6.1) are frame homomorphisms. Therefore, for each $L$-topological space $(X, \tau)$, the system $\mathcal{O}(X, \tau)=\left(l_{\alpha}^{\mathcal{O}}: \mathcal{O}^{u} \equiv \tau \rightarrow \mathcal{O}^{l} \equiv l_{L}^{T}(\tau)\right)_{\alpha \in L_{1}}$ is an $L$-frame. This is the motivating example for the notion of $L$-valued frame (cf. [26]).

Further, for every $L$-continuous map $f:\left(X, \tau_{1}\right) \rightarrow\left(Y, \tau_{2}\right)$, the pair $\mathcal{O}(f)=\left(\mathcal{O}^{\mathfrak{u}}(f), \mathcal{O}^{\mathrm{l}}(f)\right)$, with $\mathcal{O}^{\mathfrak{u}}(f)(b)=$ $f^{\leftarrow}(b)$ and $\mathcal{O}^{\mathrm{l}}(f)(B)=f^{-1}(B)$, for all $b \in L^{Y}$ and $B \in 2^{Y}$, is an $L$-frame homomorphism. This defines a contravariant functor $\mathcal{O}: L$-Top $\rightarrow L$-Frm. There are also the $L$-valued spectrum functor $\Sigma: L$-Frm $\rightarrow$ $L$-Top, right adjoint to $\mathcal{O}$, and the lower forgetful functor $\imath_{L}^{F}: L$-Frm $\rightarrow$ Frm (cf. [26] for the details), such that the diagram

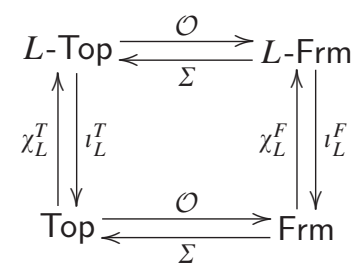

commutes.

When $L=2$, an $L$-frame is just one frame homomorphism $\varphi_{0}^{\mathcal{M}}$ which, by conditions (2) and (3), must be an isomorphism. So, an $L$-valued frame stands for a pair of (possibly distinct) isomorphic frames $\left(\mathcal{M}^{\mathfrak{u}}, \mathcal{M}^{\mathfrak{l}}\right)$ and each $L$-valued frame homomorphism is a pair of frame morphisms $\left(h^{\mathfrak{l}}, h^{\mathrm{I}}\right)$ such that each one factors through the other via an isomorphism. Therefore, the category 2-Frm is equivalent to Frm. Indeed, 2-Frm is the functor category Frm ${ }^{2}$ (where 2 is the category with 2 objects $\{u, l\}$ and an isomorphism $u \rightarrow l)$, and the latter category is clearly equivalent to Frm via functors $F:$ Frm $\rightarrow \mathrm{Frm}^{2}$ and $G: \mathrm{Frm}^{2} \rightarrow$ Frm defined by $F(M)=(M, M)$ with $\varphi_{0}^{M}=\mathrm{id}_{M}, F(h)=(h, h)$, $G\left(M^{\mathfrak{u}}, M^{\mathfrak{l}}\right)=M^{\mathfrak{u}}$ and $G\left(h^{\mathfrak{u}}, h^{\mathrm{l}}\right)=h^{\mathfrak{u}}$.

\section{The uniform crisp modification of a covering $L$-valued uniform space}

Let $L$ be a linearly ordered complete lattice. This is an integral quantale with $*=\wedge$. Let $(X, \mathfrak{u})$ be a covering $L$-uniform space. For each $\mathcal{A} \in \mathfrak{U}$ and $\alpha \in L_{1}$ let

$$
l_{\alpha}(\mathcal{A})=\left\{l_{\alpha}(a) \mid a \in \mathcal{A}\right\} .
$$

We state without proof some basic facts satisfied by the maps $\left\{l_{\alpha}: \alpha \in L_{1}\right\}$ : 
Remark 7.1. For every $\mathcal{A} \subset L^{X}, f: X \rightarrow Y$ and $\alpha, \beta \in L_{1}$, we have:

(1) $l_{\alpha}(\bigvee \mathcal{A})=\bigcup_{a \in \mathcal{A}} l_{\alpha}(a)$

(2) $l_{\alpha}(\bigwedge \mathcal{A})=\bigcap_{a \in \mathcal{A}} l_{\alpha}(a)$ if $\mathcal{A}$ is finite.

(3) $\alpha \leqslant \beta \Rightarrow l_{\beta}(a) \subseteq l_{\alpha}(a)$.

(4) $f^{-1}\left(l_{\alpha}(b)\right)=l_{\alpha}\left(f^{\leftarrow}(b)\right)$.

We also have the following:

Proposition 7.2. Let $\mathcal{A}, \mathcal{B} \in \mathfrak{U}$ and $\alpha \in L_{1}$. Then:

(1) $l_{\alpha}(\mathcal{A})$ is a cover of $X$.

(2) If $\mathcal{A} \preccurlyeq \mathcal{B}$ then $l_{\alpha}(\mathcal{A}) \preccurlyeq l_{\alpha}(\mathcal{B})$. Hence $\imath_{\alpha}(\mathcal{A} \wedge \mathcal{B}) \preccurlyeq l_{\alpha}(\mathcal{A})$ and $l_{\alpha}(\mathcal{A} \wedge \mathcal{B}) \preccurlyeq l_{\alpha}(\mathcal{B})$.

(3) $\operatorname{st}\left(l_{\alpha}(b), l_{\alpha}(\mathcal{A})\right) \subseteq l_{\alpha}(\operatorname{st}(b, \mathcal{A}))$.

(4) If $\operatorname{st}(\mathcal{A}) \preccurlyeq \mathcal{B}$ then $\operatorname{st}\left(l_{\alpha}(\mathcal{A})\right) \preccurlyeq l_{\alpha}(\mathcal{B})$.

Proof. (1) Since $\bigvee \mathcal{A}=1_{X}$ then, for each $\alpha \in L_{1}$ and each $x \in X$ there exists $a \in \mathcal{A}$ such that $a(x)>\alpha$. Consequently, $\bigcup_{a \in \mathcal{A}}\{x \in X \mid a(x)>\alpha\}=X$, that is, $\bigcup_{a \in \mathcal{A}} l_{\alpha}(a)=X$.

(2) It is straightforward.

(3) It follows from 7.1(1).

(4) For any $a \in \mathcal{A}$, let $\operatorname{st}\left(l_{\alpha}(a), l_{\alpha}(\mathcal{A})\right) \in \operatorname{st}\left(l_{\alpha}(\mathcal{A})\right)$ and $b \in \mathcal{B}$ satisfying $\operatorname{st}(a, \mathcal{A}) \leqslant b$. By property (1) of 7.1 and previous (3) one has $\operatorname{st}\left(l_{\alpha}(a), l_{\alpha}(\mathcal{A})\right) \subseteq l_{\alpha}(\operatorname{st}(a, \mathcal{A})) \subseteq l_{\alpha}(b)$.

It follows immediately from Proposition 7.2 that:

Corollary 7.3. The family $\left\{\imath_{\alpha}(\mathcal{A}) \mid \mathcal{A} \in \mathfrak{U}, \alpha \in L_{1}\right\}$ is a base for a uniformity ${ }_{L}^{U}(\mathfrak{H})$ on $X$.

Proposition 7.4. For any $f:(X, \mathfrak{U}) \rightarrow(Y, \mathfrak{B})$ in $L$-Unif, $f:\left(X, \imath_{L}^{U}(\mathfrak{U})\right) \rightarrow\left(Y, l_{L}^{U}(\mathfrak{B})\right)$ is a morphism of Unif.

Proof. For each $\mathcal{B} \in \mathfrak{B}$ and $\alpha \in L_{1}, f^{-1}\left[l_{\alpha}(\mathcal{B})\right]=\left\{f^{-1}\left(l_{\alpha}(b)\right) \mid b \in \mathcal{B}\right\}$ and $f^{-1}\left(l_{\alpha}(b)\right)=l_{\alpha}\left(f^{\leftarrow}(b)\right)$ (Remark 7.1). By hypothesis, $f^{-1}[\mathcal{B}]=\left\{f^{\leftarrow}(b) \mid b \in \mathcal{B}\right\} \in \mathfrak{U}$, so $l_{\alpha}\left(f^{-1}[\mathcal{B}]\right)=\left\{l_{\alpha}\left(f^{\leftarrow}(b)\right) \mid b \in \mathcal{B}\right\} \in l_{L}^{U}(\mathfrak{H})$, which shows that $f^{-1}\left[\imath_{\alpha}(\mathcal{B})\right] \in \imath_{L}^{U}(\mathfrak{H})$ as required.

Thus, we have a functor $\imath_{L}^{U}: L$-Unif $\rightarrow$ Unif such that $\imath_{L}^{U} \cdot \chi_{L}^{U}=$ idUnif. Recall the forgetful functor $F_{3}: L$-Unif $\rightarrow$ $L$-Top from Section 3. We have:

Proposition 7.5. $F_{1} \cdot l_{L}^{U}=l_{L}^{T} \cdot F_{3}$.

Proof. For objects we need to show that $F_{1}\left(\imath_{L}^{U}(X, \mathfrak{U})\right)=\imath_{L}^{T}\left(F_{3}(X, \mathfrak{u})\right)$ for every $L$-uniform space $(X, \mathfrak{u})$, that is, $\imath_{L}^{T}\left(\tau_{\mathfrak{l}}\right)$ is precisely the topology $\mathcal{T}_{l_{L}^{U}(\mathfrak{l})}$ induced by the crisp uniformity $\imath_{L}^{U}(\mathfrak{U} \mathfrak{l}) . \mathcal{T}_{l_{L}^{U}(\mathfrak{l})} \subseteq \imath_{L}^{T}\left(\tau_{\mathfrak{l}}\right):$ Let $A \in \mathcal{T}_{l_{L}^{U}(\mathfrak{l} \mathfrak{l})}$. Then for every $x \in A$ there exists $l_{\alpha_{x}}\left(\mathcal{A}_{x}\right) \in \imath_{L}^{U}(\mathfrak{l})$ such that $\operatorname{st}\left(\{x\}, l_{\alpha_{x}}\left(\mathcal{A}_{x}\right)\right) \subseteq A$. Therefore,

$$
A \supseteq \bigcup_{x \in A} \operatorname{st}\left(\{x\}, l_{\alpha_{x}}\left(\mathcal{A}_{x}\right)\right)=\bigcup_{x \in A}\left(\bigcup\left\{l_{\alpha_{x}}(a) \mid a \in \mathcal{A}_{x}, x \in l_{\alpha_{x}}(a)\right\}\right) .
$$

Since the reverse inclusion is obvious, we have $A=\bigcup_{x \in A} \operatorname{st}\left(\{x\}, l_{\alpha_{x}}\left(\mathcal{A}_{x}\right)\right) \in \imath_{L}^{T}\left(\tau_{\mathfrak{U}}\right)$.

$l_{L}^{T}\left(\tau_{\mathfrak{l}}\right) \subseteq \mathcal{T}_{l_{L}^{U}(\mathfrak{l})}$ : Now let $l_{\alpha}(a) \in l_{L}^{T}\left(\tau_{\mathfrak{l}}\right)$ and $x \in l_{\alpha}(a)$. Then $a(x)>\alpha$. Since $a \in \tau_{\mathfrak{l}}$, there exists $b \in L^{X}$ with $x \in l_{\alpha}(b)$ and $\operatorname{st}(b, \mathcal{A}) \leqslant a$, for some $\mathcal{A} \in \mathfrak{U}$. Hence $\imath_{\alpha}(\operatorname{st}(b, \mathcal{A})) \subseteq \imath_{\alpha}(a)$. Also, since $x \in l_{\alpha}(b)$, we have

$$
x \in \operatorname{st}\left(\{x\}, l_{\alpha}(\mathcal{A})\right) \subseteq \operatorname{st}\left(l_{\alpha}(b), l_{\alpha}(\mathcal{A}) \subseteq l_{\alpha} \operatorname{st}(b, \mathcal{A}) \subseteq l_{\alpha}(a) .\right.
$$

For morphisms the proof is straightforward. 
In conclusion, both squares in the following diagram commute:

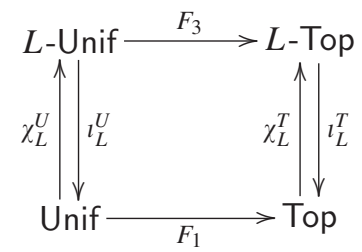

\section{The missing vertex: $L$-valued uniform frames}

Let $L$ be a linearly ordered complete lattice. For each covering $L$-valued uniform space $(X, \mathfrak{H})$, the pair $\left(X, \tau_{\mathfrak{U}}\right)$ is an $L$-topological space (Proposition 3.4) and, consequently,

$$
\left(l_{\alpha}: \tau_{\mathfrak{U}} \rightarrow \imath_{L}^{T}\left(\tau_{\mathfrak{l}}\right)\right)_{\alpha \in L_{1}}
$$

is an $L$-frame. How can we uniformize it in a canonical way?

Since $\tau_{\mathfrak{U}}$ is a subframe of $L^{X}$, it cannot be uniformized by a frame uniformity (otherwise, the lattice $L$ of values would be also uniformizable and then, as pointed out in the Introduction, $L$ would necessarily be the two-element chain (2). On the other hand, the crisp topology $\imath_{L}^{T}\left(\tau_{\mathfrak{U}}\right)$ being completely regular (by Proposition 7.5), since $\imath_{L}^{T}\left(\tau_{\mathfrak{U}}\right)=\mathcal{T}_{l_{L}^{U}}(\mathfrak{l})$ ), it is uniformizable. This justifies the introduction of uniform structures on $L$-valued frames

$$
\mathcal{M}=\left(\varphi_{\alpha}^{\mathcal{M}}: \mathcal{M}^{\mathfrak{u}} \rightarrow \mathcal{M}^{\mathfrak{l}}\right)_{\alpha \in L_{1}}
$$

as frame uniformities on the lower frame $\mathcal{M}^{\mathrm{I}}$. We say that $(\mathcal{M}, \mathcal{C})$ is an L-valued uniform frame if

$$
\mathcal{M}=\left(\varphi_{\alpha}^{\mathcal{M}}: \mathcal{M}^{\mathfrak{u}} \rightarrow \mathcal{M}^{\mathrm{I}}\right)_{\alpha \in L_{1}}
$$

is an $L$-valued frame and $\mathcal{C}$ is a frame uniformity on the lower frame $\mathcal{M}^{\mathrm{I}}$. An $L$-valued uniform homomorphism $h:(\mathcal{M}, \mathcal{C}) \rightarrow(\mathcal{N}, \mathcal{D})$ is a pair

$$
\left(h^{\mathfrak{u}}: \mathcal{M}^{\mathfrak{u}} \rightarrow \mathcal{N}^{\mathfrak{u}}, h^{\mathfrak{l}}:\left(\mathcal{M}^{\mathfrak{l}}, \mathcal{C}\right) \rightarrow\left(\mathcal{N}^{\mathfrak{l}}, \mathcal{D}\right)\right)
$$

with $h^{\mathfrak{u}}$ a frame homomorphism and $h^{\mathfrak{I}}$ a uniform frame homomorphism satisfying $h^{\mathfrak{I}} \cdot \varphi_{\alpha}^{\mathcal{M}}=\varphi_{\alpha}^{\mathcal{N}} \cdot h^{\mathfrak{u}}$ for every $\alpha \in L_{1}$.

In the case $L=2$, an $L$-valued uniform frame $(\mathcal{M}, \mathcal{C})$ is just a frame isomorphism $\varphi_{0}^{\mathcal{M}}: \mathcal{M}^{\mathfrak{u}} \rightarrow\left(\mathcal{M}^{\mathrm{I}}, \mathcal{C}\right)$ and an $L$-valued uniform homomorphism is a pair of frame morphisms $\left(h^{\mathfrak{u}}, h^{\mathfrak{l}}\right)$, the latter one being uniform, such that each one factors through the other via an isomorphism. So, the category 2-UFrm is clearly equivalent to UFrm via functors $F:$ UFrm $\rightarrow$ 2-UFrm and $G: 2$-UFrm $\rightarrow$ UFrm defined by $F(\mathcal{M}, \mathcal{C})=\left(\varphi_{0}^{\mathcal{M}}: \mathcal{M} \rightarrow(\mathcal{M}, \mathcal{C})\right)$ with $\varphi_{0}^{\mathcal{M}}=\mathrm{id}_{\mathcal{M}}$, $F(h)=(h, h), G\left(\varphi_{0}^{\mathcal{M}}: \mathcal{M}^{\mathfrak{u}} \rightarrow\left(\mathcal{M}^{\mathrm{l}}, \mathcal{C}\right)\right)=\left(\mathcal{M}^{\mathrm{l}}, \mathcal{C}\right)$ and $G\left(h^{\mathfrak{u}}, h^{\mathrm{I}}\right)=h^{\mathrm{I}}$.

Then, for each covering $L$-valued uniform space $(X, \mathfrak{l})$, we have

$$
\mathcal{O}_{L}^{U}(X, \mathfrak{U})=\left(l_{\alpha}: \tau_{\mathfrak{U}} \rightarrow l_{L}^{T}\left(\tau_{\mathfrak{U}}\right)\right)_{\alpha \in L_{1}} \in L-\text { Frm }
$$

In order to establish the open functor $\mathcal{O}: L$-Unif $\rightarrow L$-UFrm we need to endow the crisp topology $l_{L}^{T}\left(\tau_{\mathfrak{U}}\right)$ of $\left(X, \tau_{\mathfrak{U}}\right)$ with a canonical frame uniformity. This is possible because, as remarked above, the topology $\imath_{L}^{T}\left(\tau_{\mathfrak{U}}\right)$ is always completely regular.

So, let $\mathfrak{U}_{\tau_{\mathfrak{U}}}$ be the collection of all $\tau_{\mathfrak{U}}$-open $L$-covers in $\mathfrak{U}$ (i.e. all $\mathcal{A} \in \mathfrak{U}$ such that $\mathcal{A} \subseteq \tau_{\mathfrak{U}}$ ). Each $\mathcal{A} \in \mathfrak{U}_{\tau_{\mathfrak{V} \mathfrak{I}}}$ is a cover of the spatial frame $\tau_{\mathfrak{U}}$. For each $\mathcal{A} \in \mathfrak{U}_{\tau_{\mathfrak{U}}}$ and $\alpha \in L_{1}$ let $l_{\alpha}(\mathcal{A})=\left\{l_{\alpha}(a) \mid a \in \mathcal{A}\right\}$. Then, by Remark 7.1(1), $l_{\alpha}(\mathcal{A})$ is a cover of $\imath_{L}^{T}\left(\tau_{\mathfrak{U}}\right)$.

Proposition 8.1. For every covering L-uniform space $(X, \mathfrak{H}),\left\{l_{\alpha}(\mathcal{A}) \mid \alpha \in L_{1}, \mathcal{A} \in \mathfrak{U}_{\tau_{\mathfrak{U}}}\right\}$ is a base for a frame uniformity $\mathcal{C}_{\mathfrak{U}}$ on $\imath_{L}^{T}\left(\tau_{\mathfrak{U}}\right)$.

Proof. It remains to show the star-refinement condition, since (U3) is equivalent to the regularity of $\imath_{L}^{T}\left(\tau_{\mathfrak{U}}\right)$. This is a consequence of 7.2(4), since $\operatorname{st}(\mathcal{B}) \preccurlyeq \mathcal{A}$ implies $\operatorname{st}\left(l_{\alpha}(\mathcal{B})\right) \preccurlyeq l_{\alpha}(\mathcal{A})$ for every $\alpha \in L_{1}$. 
It is now easy to check that the correspondence

$$
(X, \mathfrak{H}) \longmapsto\left(l_{\alpha}: \tau_{\mathfrak{U}} \rightarrow\left({ }^{T}\left(\tau_{\mathfrak{U}}\right), \mathcal{C}_{\mathfrak{U}}\right)\right)_{\alpha \in L_{1}}
$$

is functorial and establishes a functor $\mathcal{O}: L$-Unif $\rightarrow L$-UFrm. Conversely, following the lines of [26], it is not hard to construct the $L$-valued version $\Sigma: L$-UFrm $\rightarrow L$-Unif of the well-known spectrum functor $\Sigma: U F r m \rightarrow$ Unif, right adjoint to $\mathcal{O}$, and the uniform version $\imath_{L}^{U F}: L$-UFrm $\rightarrow$ UFrm of the lower forgetful functor $\imath_{L}^{F}$, that make the diagram

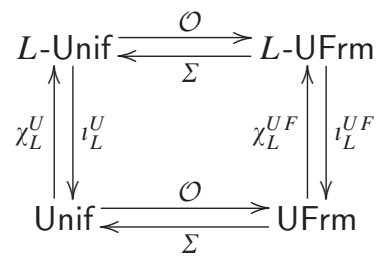

commutative.

Finally, consider the forgetful functor $F_{4}: L$-UFrm $\rightarrow L$-Frm forgetting the uniform structure. Putting the functors here considered altogether we obtain the desired commutative cube for a complete chain $L$ :

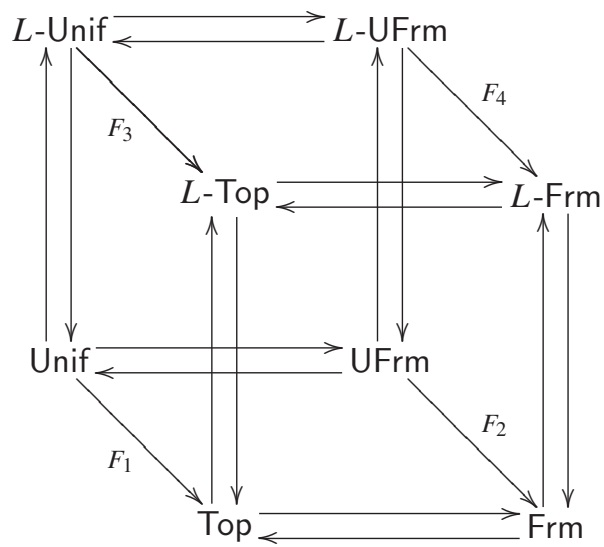

\section{Acknowledgments}

The first, second, and fourth authors acknowledge financial support from the Ministry of Education and Science of Spain and FEDER under Grant MTM2006-14925-C02-02 and from the University of the Basque Country under Grant UPV05/101. The third author acknowledges financial support from the Centre of Mathematics of the University of Coimbra/FCT.

\section{References}

[1] J. Adámek, H. Herrlich, G.E. Strecker, Abstract and Concrete Categories, Wiley Interscience Pure and Applied Mathematics, Wiley, New York, 1990.

[2] B. Banaschewski, A. Pultr, Samuel compactification and completion of uniform frames, Math. Proc. Cam. Philos. Soc. 108 (1990) 63-78.

[3] G. Birkhoff, Lattice Theory, third ed., AMS Colloquium Publications, 1967.

[4] C.L. Chang, Fuzzy topological spaces, J. Math. Anal. Appl. 24 (1968) 182-190.

[5] M. Erné, J. Koslowski, A. Melton, G.E. Strecker, A primer on Galois connections, in: Papers on General Topology and Applications, Madison, WI, 1991, Ann. New York Acad. Sci. 704 (1993) 103-125.

[6] M.J. Ferreira, J. Picado, The Galois approach to uniform structures, Quaestiones Math. 28 (2005) 355-373.

[7] P. Fletcher, W. Hunsaker, Entourage uniformities for frames, Monatsh. Math. 112 (1991) 271-279.

[8] P. Fletcher, W. Hunsaker, W. Lindgren, A functional approach to uniform and quasi-uniform frames, in: Topology with Applications, Bolyai Society Mathematical Studies, Vol. 4, Szekszárd, 1993, pp. 217-222.

[9] J. Gutiérrez García, M.A. de Prada Vicente, Fuzzy t-uniformidades, Publ. Sem. Mat. García de Galdeano Ser. I 3 (1992) 1-14.

[10] J. Gutiérrez García, M.A. de Prada Vicente, Super uniform spaces, Quaestiones Math. 20 (1997) 291-309. 
[11] J. Gutiérrez García, M.A. de Prada Vicente, A. Šostak, A unified approach to the concept of fuzzy, in: S.E. Rodabaugh, E.P. Klement (Eds.), Topological and Algebraic Structures in Fuzzy Sets, Trends Log. Stud. Log. Libr., Vol. 20, Kluwer Academic Publishers, Dordrecht, 2003, pp. $81-114$.

[12] U. Höhle, Commutative residuated L-monoids, in: U. Höhle, E.P. Klement (Eds.), Non-classical Logics and their Applications to Fuzzy Subsets, Kluwer Academic Publishers, Dordrecht, 1995, pp. 53-106.

[13] U. Höhle, Locales and L-topologies, Mathematik-Arbeitspapiere, Vol. 48, University of Bremen, 1997, pp. $223-250$.

[14] U. Höhle, A. Šostak, Axiomatic foundations of fixed-basis fuzzy topology, Mathematics of Fuzzy Sets: Logic, Topology, and Measure Theory, The Handbooks of Fuzzy Sets Series, Vol. 3, Kluwer Academic Publishers, Dordrecht, 1999, pp. 123-272.

[15] B. Hutton, Uniformities on fuzzy topological spaces, J. Math. Anal. Appl. 58 (1977) 559-571.

[16] J. Isbell, Atomless parts of spaces, Math. Scand. 31 (1972) 5-32.

[17] P.T. Johnstone, Stone Spaces Cambridge Studies in Advanced Mathematics, Vol. 3, Cambridge University Press, Cambridge, 1982.

[18] T. Kubiak, The topological modification of the L-fuzzy unit interval, in: S.E. Rodabaugh, E.P. Klement, U. Höhle (Eds.), Applications of Category Theory to Fuzzy Subsets, Theory Decis. Lib. Ser. B Math. Statist. Methods, Vol. 14, Kluwer Academic Publishers, Dordrecht, 1992, pp. 275-305.

[19] R. Lowen, Fuzzy topological spaces and fuzzy compactness, J. Math. Anal. Appl. 56 (1976) 621-633.

[20] R. Lowen, Fuzzy uniform spaces, J. Math. Anal. Appl. 82 (1981) 370-385.

[21] J. Picado, Structured frames by Weil entourages, Appl. Categ. Struct. 8 (2000) 351-366.

[22] J. Picado, The quantale of Galois connections, Algebra Universalis 52 (2004) 527-540.

[23] J. Picado, A. Pultr, A. Tozzi, Locales, in: M.C. Pedicchio, W. Tholen (Eds.), Categorical Foundations-Special Topics in Order Topology Algebra and Sheaf Theory, Encyclopedia of Mathematics and its Applications, Vol. 97, Cambridge University Press, Cambridge, 2003, pp. $49-101$.

[24] M.A. de Prada Vicente, M. Macho Stadler, $t$-Prefilter theory, Fuzzy Sets and Systems 38 (1990) 115-124.

[25] A. Pultr, Pointless uniformities I. Complete regularity, Comment. Math. Univ. Carolin. 25 (1984) 91-104.

[26] A. Pultr, S.E. Rodabaugh, Lattice-valued frames functor categories and classes of sober spaces, in: S.E. Rodabaugh, E.P. Klement (Eds.), Topological and Algebraic Structures in Fuzzy Sets, Trends Log. Stud. Log. Libr., Vol. 20, Kluwer Academic Publishers, Dordrecht, 2003, pp. $153-187$.

[27] A. Pultr, S.E. Rodabaugh, Category theoretic aspects of chain-valued frames: part I: categorical and presheaf theoretic foundations, Fuzzy Sets and Systems 159 (2008) 501-528.

[28] A. Pultr, S.E. Rodabaugh, Category theoretic aspects of chain-valued frames: part II: applications to lattice-valued topology, Fuzzy Sets and Systems 159 (2008) 529-558.

[29] S.E. Rodabaugh, A theory of fuzzy uniformities with applications to the fuzzy real lines, J. Math. Anal. Appl. 129 (1988) $37-70$.

[30] S.E. Rodabaugh, Powerset operator based foundation for point-set lattice-theoretic (POSLAT) fuzzy set theories and topologies, Quaestiones Math. 20 (1997) 463-530.

[31] S.E. Rodabaugh, Separation axioms: representation theorems, compactness, and compactifications, in: U. Höhle, S.E. Rodabaugh (Eds.), Mathematics of Fuzzy Sets: Logic, Topology, and Measure Theory, The Handbooks of Fuzzy Sets Series, Vol. 3, Kluwer Academic Publishers, Boston/Dordrecht/London, 1999, pp. 481-552.

[32] S.E. Rodabaugh, Axiomatic foundations for uniform operator quasi-uniformities, in: S.E. Rodabaugh, E.P. Klement (Eds.), Topological and Algebraic Structures in Fuzzy Sets, Trends Log. Stud. Log. Libr., Vol. 20, Kluwer Academic Publishers, Dordrecht, 2003 , pp. 199-233.

[33] J. Tukey, Convergence and Uniformity in Topology, Ann. Math. Studies, Vol. 1, Princeton University Press, Princeton, NJ, 1940.

[34] A. Weil, Sur les Espaces à Structure Uniforme et sur la Topologie Générale, Act. Sci. et Ind., Vol. 551, Hermann Press, Paris, 1937.

[35] P. Wuyts, On the determination of F.T.S and F.N.S by their level topologies, Fuzzy Sets and Systems 12 (1984) $71-85$.

[36] P. Wuyts, On level topologies and maximality of F.T.S, Fuzzy Sets and Systems 79 (1996) 337-339.

[37] P. Wuyts, R. Lowen, E. Lowen, Reflectors and Correflectors in F.T.S, Comp. Math. Appl. 16 (1988) 823-836. 